\title{
ALGUNS ASPECTOS DA APLICACÃO DA SEGUNDA LEI DE MITSCHERLICH E DA EQUAÇÃO DE REGRESSÃO QUADRÁTICA A MATURAÇÃO DA CANA-DE-AÇÚCAR
}

\author{
ROMEU MAGNANI
}

Orientador: Dr. FREDERICO PIMENTEL GOMES

Dissertação apresentada à Escola Superior de Agricultura "Luiz de Queiroz", da Universidade de São Paulo, para obtenção do Título de Mestre em Agronomia - Área de Concentração: Estatística e Experimentação Agronômica.

\footnotetext{
$P|R A C| C A B A$

Estado de São Paulo - Brasil

Fevereiro - 1985
} 
ii.

Dedico este trabalho à Diná

e às nossas filhas: Flávia, Cristina e Marina. 


\section{AGRADEC IMENTOS}

- Ao Dr. Frederico Pimentel Gomes, pela orien tação segura e valiosa na realização deste trabalho.

- Ao Dr. Humberto de Campos, Coordenador do Curso de Pós-Graduação em Estatística e Experimentação Agronômica, pelo apoio.

- Aos Professores do Curso de Pós-Graduação, pelos ensinamentos transmitidos.

- Ao Prof. Dalton Geraldo Guaglianoni, especial amigo e companheiro de luta.

- Aos colegas do Curso de Pós-Graduação, pelo convívio.

- Aos funcionários do Departamento de Matemática e Estatística da ESALQ: Maria IzalinaFerreiraAlves, Ro sa Maria Alves e Octávio Frassetto, pela amizade.

- Aos colegas do Departamento de Físico-Quími ca do Instituto de Química de Araraquara, pelasolidariedade.

- Aos Professores: Celi Vasques Crepaldi, João Sahão Jr. e José Maria Lopes, pela amizade, incentivo e cola boração .

- Ao Prof. José Arana Varella, pela versão do resumo para o inglês.

- A todos que, direta ou indiretamente, con tribuiram na execução deste trabalho. 
INDICE

Página

RESUMO $\ldots \ldots \ldots \ldots \ldots \ldots \ldots \ldots \ldots \ldots \ldots \ldots \ldots \ldots \ldots \ldots$

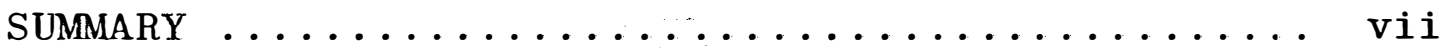

1. INTRODUÇÃ̃o ......................... 1

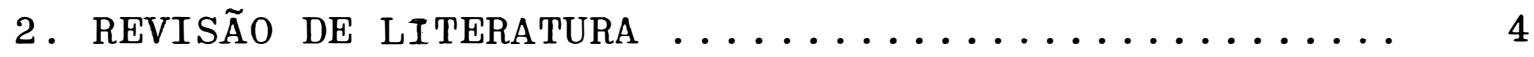

3. MATERIAL E MEtodos $\ldots \ldots \ldots \ldots \ldots \ldots \ldots \ldots \ldots \ldots \ldots$

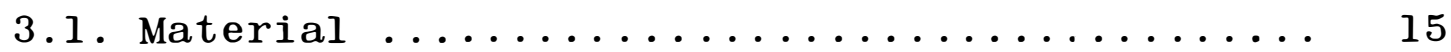

3.2. Método dos Quadrados Mínimos .......... 16

3.2.1. Modelo da Regressão Quadrática ...... 16

3.2.2. Estimação dos Parâmetros .......... 17

3.2.3. Análise de Variância ............ 18

3.2.4. Programą̧ão do Método ............ 21

3.3. Métodos do Gradiente ................ 24

3.3.1. Modelo da Segunda Lei de Mitscherlich 24

3.3.2. Método de Newton .............. 27

3.3.3. Método de Gauss ............... 29

3.3.4. Método de Marquardt ............ 32

3.3.5. Programação dos Métodos do Gradiente. 33

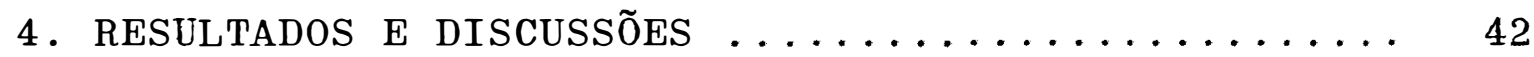

4.1. Estimativas dos Parâmetros do Modelo Quadráti

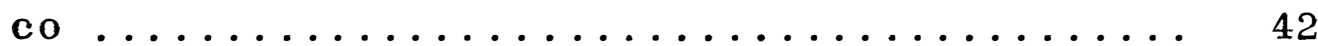

4.2. Estimativas dos Parâmetros da Segunda Lei de Mitscherlich .................. 45

4.3. Análise dos Ajustamentos ............. 56

5. CONCLUSÕES .........................61

6. Literatura CItAdA $\ldots \ldots \ldots \ldots \ldots \ldots \ldots \ldots \ldots \ldots \ldots \ldots \ldots$

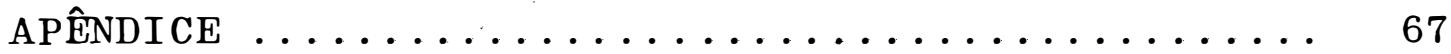




\author{
ALGUNS ASPECTOS DA APLICAÇÃO DA SEGUNDA LEI \\ DE MITSCHERLICH E DA EQUAÇÃO DE REGRESSÃO \\ QUADRATTICA A MATURAÇÃO DA CANA-DE-AÇỨCAR \\ Autor: Romeu Magnani \\ Orientador: Frederico Pimentel Gomes
}

RESUMO

Certos fenômenos, nos quais uma variável mani festa um crescimento inicial, em função de outra variável, e em seguida um decrescimento, tal como a quantidade de sacaro se na cana-de-açúcar conforme esta se desenvolve, têm sido estudados pelo modelo de regressão Quadrática principalmente pela facilidade da estimação dos parâmetros, ainda que a rea lidade não mostre simetria em relação ao eixo vertical que passa pelo ponto de máximo. Por outro lado, o modelo da Segun da Lei de Mitscherlich pode dar uma descrição mais verdadeira, porém a estimação dos parâmetros é difícil.

Neste trabalho, para 27 grupos de dados de ma turação da cana-de-açúcar, expressos em Pol\% da cana, com o apoio de um microcomputador pessoal, é estudada a estimação dos parâmetros da Segunda Lei de Mitscherlich pelos métodos de Newton, Gauss e Marquardt; são analisados os dois ajusta- 
mentos pelos testes estatísticos e os algoritmos de cálculo são codificados em linguagem BASIC.

Dos métodos estudados, o mais eficiente foi o de Marquardt, que permitiu a superação de dificuldades na con vergência, decorrentes da localização do ponto onde se inicia o processo iterativo. Não foi possível estabelecer, por critérios exclusivamente estatísticos, o melhor ajustamento. En tretanto, um exame dos resultados evidenciou que a maturação da cana-de-açúcar, quanto à quantidade de Pol\% da cana, pode ser estudada até o máximo por um modelo ou outro, mas, a par tir daí, a Segunda Lei de Mitscherlich acompanha melhor a ten dência dos dados experimentais, fornecendo uma visão global mais real do fenômeno em questão e, portanto, mais informativa. 
vii.

SOME ASPECTS OF THE MITSCHERLICH'S SECOND LAW AND THE QUADRATIC REGRESSION EQUATION APPLICATION ON THE SUGAR CANE MATURATION

Author: Romeu Magnani

Adviser: Frederico Pimentel Gomes

SUMMARY

Some phenomena, in which a variable initially increases and then decreases as a function of another variable, have been studied using the quadratic regression model. Although these phenomena follow well the model up to the maximum, from this point on the curve decreases more smoothly as predicted by the model. This is the case of the sugar cane maturation. Mitscherlich's Second Regression Law can give a better descrip tion but the parameter estimation is more dificult.

This work studies the maturation in 27 groups of data on the maturation of sugar cane, by measuring the quan tity of saccarose (in Pol\% of cane). The parameters of Mitscherlich's Second Law were estimated by Newton, Gauss and Marquardt methods. Both regression equations (quadratic and Mitscherlich's) were analyzed by statistical tests.

The Marquardt method was more efficient to es 
viii.

timate the parameters of the Mitscherlich's Law. This method leads easily to convergence. It was concluded that the statis tical tests are not adequate as indicative of the best adjus ment. However, the results showed that Mitscherlich's Law gives the best insight of the phenomena studied. 
1. INTRODUÇÃO

Até recentemente, trabalhos que necessitassem, para o seu desenvolvimento, do auxílio de um computador, encontravam dificuldades. Hoje, devido ao advento dos microcom putadores, os quais possuem incorporada uma linguagem de uso e aprendizado fácil, o BASIC, o pesquisador começa a ter à sua disposição essa poderosa ferramenta de trabalho, onde ele mesmo pode operar. Num futuro próximo isso deverá servir tam bém ao estudante em sala de aula. Assim, modelos matemáticos para descrever experimentos e os correspondentes métodos de estimação dos parâmetros, não adotados em razão da complexidade nos cálculos, devem ser repensados e reestudados comvis ta a substituirem aqueles utilizados principalmente pela sim plicidade que manifestam.

Uma equação para a qual o computador é instru mento indispensável de apoio é a Segunda Lei de Mitscherlich, 


$$
Y=b_{1}\left[1-10^{-b_{3}\left(X+b_{2}\right)}\right] 10^{-b_{4}\left(X+b_{2}\right)^{2}}
$$

com $\mathrm{X}$ e $\mathrm{Y}$ as variáveis independente e dependente respectivamente; $\mathrm{b}_{1}, \mathrm{~b}_{2}, \mathrm{~b}_{3} \mathrm{e} \mathrm{b}_{4}$ os parâmetros a serem estimados através de dados experimentais, e sua curva representativa teria o aspecto da figura abaixo, para $b_{4}$ positivo e os parâmetros $\mathrm{b}_{1}$ e $\mathrm{b}_{3}$, em geral, positivos:

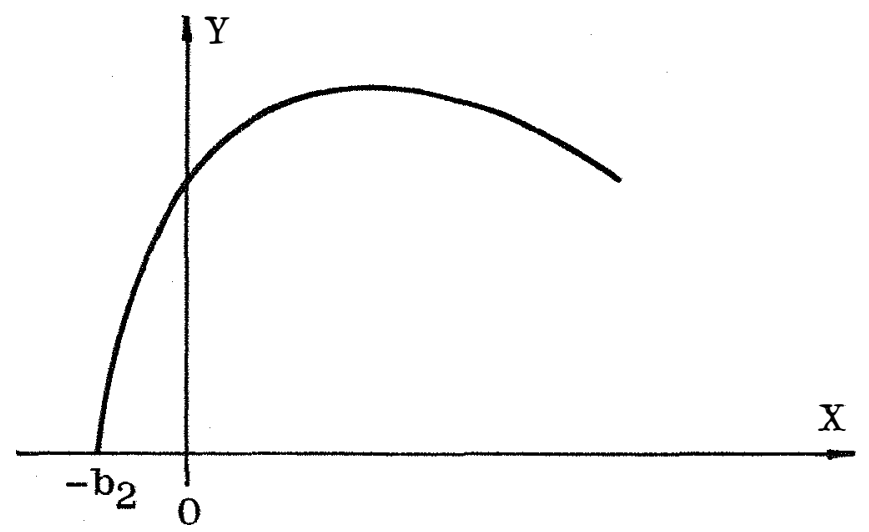

Esta lei pode, em princípio, descrever fenômenos nos quais, enquanto $X$ cresce, há um crescimento inicial rápido de $Y$ até atingir um máximo e, a partir daí, surge um decrescimento menos in tenso. Revelam tal comportamento, por exemplo, as produções de culturas submetidas a doses crescentes de um nutriente,ou os teores de sacarose na cana-de-açúcar, medidos conforme a cana se desenvolve. Nestes casos, e principalmente noúltimo, tem sido aplicado o modelo de regressão Quadrática, de equação

$$
\mathrm{Y}=\mathrm{b}_{0}+\mathrm{b}_{1} \mathrm{X}+\mathrm{b}_{2} \mathrm{X}^{2}
$$

por ser mais simples e responder satisfatoriamente, pelo menos 
até que o máximo seja atingido.

Este trabalho tem em mira focalizar alguns as pectos do ajustamento, para uma situação experimental, de um modelo considerado mais complicado, o não linear nos parâmetros, e ao mesmo tempo compará-lo com aquele mais simples, o linear. Este objetivo será seguido no caso particular da aná lise do experimento de maturação da cana-de-açúcar, pelos mo delos da Segunda Lei de Mitscherlich e Quadrático, pretenden do-se basicamente:

1ㅇ) Verificar e tirar conclusões sobre o desem penho, em um microcomputador, dos métodos iterativos de Newton, Gauss e Marquardt na estimação dos parâmetros da Segunda Lei de Mitscherlich.

2o) Sugerir programas em linguagem BASIC para os métodos adotados.

3) Analisar experimentos de maturação da cana de-açúcar pelos modelos da Segunda Lei de Mitscherlich e da regressão Quadrática, examinando a representatividade dos tes tes estatísticos usuais de modelos lineares quando aplicados indistintamente aos dois casos. 
4.

2. REVISÃO DE LITERATURA

Considerando que os objetivos propostos para o presente trabalho compreendem uma discussão sobremétodos ite rativos de estimação de parâmetros, incluindo um confronto en tre o modelo linear e o não linear quando ajustados aos mesmos dados, iniciaremos esta revisão de literatura com um pequeno exame do primeiro modelo, por demais conhecido.

SEAL (1967) apresenta uma visão histórica do desenvolvimento do modelo de regressão linear,

$$
Y_{j}=\sum_{k=1}^{m} b_{k} X_{k j}+E_{j} \quad(j=1,2, \ldots, n ; n>m)
$$

onde os $\mathrm{X}_{\mathrm{kj}}$ são "coeficientes" conhecidos (variáveis indepen dentes), os $\mathrm{Y}_{\mathrm{j}}$ são "observações" (variável dependente), os $\mathrm{b}_{\mathrm{k}}$ são as "incógnitas" (parâmetros) e os $\mathrm{E}_{\mathbf{j}}$ são os "erros", citando ter sido Legendre, em 1805, o primeiro a sugerir em pu blicação o Método dos Quadrados Mínimos para o cálculo de es 
timativas dos parâmetros, ao estabelecer o princípio de que a solução mais satisfatória para os $b_{k}$ é aquela que minimiza a soma de quadrados dos erros. Entretanto, foi Gauss, no seu Theoria Motus, em 1809, o primeiro a discutir o modelo de re gressão linear associado a uma distribuição de probabilidades dos erros. Em notação matricial o modelo fica:

$$
\mathrm{Y}=\mathrm{XB}+\mathrm{E}
$$

com X a matriz ( $\mathrm{n} \times \mathrm{m}$ ) de coeficientes, B o vetor de $\underline{\mathrm{m}}$ parâme tros, Y e E os vetores, respectivamente, de observações e er ros. O método dos quadrados mínimos fornece uma únicasolução,

$$
\hat{\mathrm{B}}=(\mathrm{X} \cdot \mathrm{X})^{-1} \mathrm{X} \cdot \mathrm{Y}
$$

desde que $X^{\prime} X$ seja não-singular, sendo $X^{\prime}$ a matriz transposta de X. A partir de hipoteses para os erros, conclusões probabilísticas a respeito dos parâmetros e do modelo são obtidas. No seu artigo, Seal discorre sobre outros autores que fornece ram substanciais contribuições, destacando, do século 19 até antes de 1950, além do próprio Gauss, Cauchy, Chebyshev, Pizzetti, Karl Pearson e R.A. Fisher. Um impulso maior, na aplicação das técnicas estatísticas para a construção e estimação de modelos matemáticos ligados a experimentos, veio dos trabalhos de G.E. Box e seus colaboradores, por volta de 1950.

Para focalizar os métodos de estimação e o es tudo de modelos não lineares,

$$
Y_{j}=f\left(X_{j} ; B\right)+E_{j}
$$


principiamos com a análise da publicação de BARD (1974), o qual considera que métodos computacionais com os nomes de Newton, Gauss e Cauchy têm sido conhecidos por muito tempo, mas suas aplicações mais amplas a problemas práticos aguarda ram o advento dos computadores eletrônicos. Bard expõe e dis cute os chamados métodos iterativos do gradiente que exigem, em cada iteração, o cálculo do gradiente da função "soma de quadrados"

$$
S(B)=\sum_{j=1}^{n}\left[Y_{j}-f\left(x_{j} ; B\right)\right]^{2}
$$

em cuja minimização estamos interessados. No início da i-ésima iteração está disponível o vetor $B_{i}=\left[b_{1 i}, b_{2 i}, \ldots, b_{m i}\right]^{\prime}$ e um novo vetor $\mathrm{B}_{i+1}$ é procurado através da fórmula

$$
\mathrm{B}_{i+1}=\mathrm{B}_{\mathbf{i}}-\mathrm{p}_{\mathbf{i}} \mathrm{R}_{\mathbf{i}} \mathrm{G}_{i}
$$

onde $p_{i}$ é um escalar, $R_{i}$ uma matriz $(\mathrm{mxm})$ e $G_{i}$ é o vetor gradiente de $S(B)$ calculados para $B=B_{i}$. Devemos notar que a direção $-G_{i}$ é aquela em que $S(B)$ decresce mais rapidamente no ponto $\mathrm{B}_{i}$ e $\mathrm{p}_{\mathbf{i}} \mathrm{R}_{\mathbf{i}}$ estabelece "quanto caminhar" nessa direção. 0 autor ressalta que os vários métodos do gradiente diferem um do outro pela escolha de $p_{i}$ e $R_{i}$, entretanto, é comumente desejável que $R_{1}$ seja definida positiva, de onde decorre que se $G_{i}$ é um vetor não nulo, então, para um número $p_{i}$ suficien te pequeno, teremos $S\left(B_{i+1}\right)<S\left(B_{i}\right)$, o que, num certo sentido, assegura que $B_{i+1}$ está mais próximo do ponto de mínimo do que $B_{i}$. Dos métodos considerados por Bard, o mais sim- 
ples, chamado "método da descida mais rápida" (Steepest Descent), admitecomo $\mathrm{R}_{\mathbf{i}}$ a matriz de identidade, mas não é recomen dado na prática. Quanto aos métodos clássicos, destaca o de Newton $\operatorname{com} p_{i}=1$ e $R_{i}=H_{i}^{-1}$, sendo $H_{i}$ a matriz Hessiana de $S(B)$ calculada para $B=B_{i}$, e o método de Gauss com $p_{i}=1$ e $R_{i}=\tilde{H}_{i}^{-1}$, admitindo $\tilde{\mathrm{H}}$, uma aproximação de $\mathrm{H}$, com elementos

$$
\tilde{h}_{k m}=2 \sum_{j=1}^{n}\left[\partial f\left(x_{j} ; B\right) / \partial b_{k}\right]\left[\partial f\left(x_{j} ; B\right) / \partial b_{m}\right] \text {. }
$$

Considera, ainda, algumas modificações introduzidas neste mé todo, propondo algoritmos para melhorá-lo. o métocio de Gauss, na forma original, consiste no desenvolvimento em série de Taylor até o termo de primeira ordem da função $f\left(x_{j} ; B\right)$ em tor no do ponto $B_{i}$, o qual, para $B_{i}$ próximo de $B$, permite escrever:

$$
f\left(x_{j} ; B\right) \simeq f\left(x_{j} ; B_{i}\right)+\sum_{k=1}^{m}\left[\partial f\left(x_{j} ; B\right) / \partial b_{k}\right]_{B=B_{i}}\left(b_{k}-b_{k i}\right)
$$

e considerando

$$
\begin{aligned}
Y_{j}^{*} & =Y_{j}-f\left(x_{j} ; B_{i}\right) \\
a_{k} & =b_{k}-b_{k i} \\
X_{k j}^{*} & =\left[\partial f\left(x_{j} ; B\right) / \partial b_{k}\right]_{B=B_{i}}
\end{aligned}
$$

o modelo torna-se aproximadamente da forma:

$$
\mathrm{Y}_{\mathrm{j}}^{*}=\sum_{\mathrm{k}=1}^{\mathrm{m}} \mathrm{a}_{\mathrm{k}} \mathrm{X}_{\mathrm{kj}}^{*}+\mathrm{E}_{\mathrm{j}} \quad(\mathrm{j}=1,2, \ldots, ; \mathrm{n}>\mathrm{m})
$$

agora, linear nos parâmetros $a_{k}$. A resolução de quadrados mí 
nimos para modelos lineares pode ser seguida, gerando a esti mativa $\hat{A}=\hat{B}-B_{i}$. Daí, se aceitarmos $\hat{B}=B_{i+1}$, vem a fórmula ite rativa:

$$
B_{i+1}=B_{i}+\hat{A}_{i}=B_{i}-\tilde{H}_{i}^{-1} G_{i}
$$

Um capítulo do livro de Bard é dedicado inteiramente à questão das conclusões, depois de obtidas as estimativas, no que concerne à validade destas, ou do modelo ajustado. Como acon tece nos textos gerais, no caso um dos mais completos sobre estimação de parâmetros em modelos não lireares, as dificuldạ des na pesquisa do mínimo são apresentadas de maneira ampla, sem entrar em detalhes para equações específicas. Além disso, são superficiais as consideraçoes sobre o problema da escolha do ponto de partida dos métodos iterativos.

Alguns artigos existentes na literatura sobre métodos não lineares serão, agora, examinados. Dentre eles destaca-se o de LEVENBERG (1944) que apresenta uma extensão do método de linearização de Gauss de modo a assegurar uma melhora na solução inicial. Considerando que o método de Gauss, ao abandonar os termos maiores do que primeira ordem no desen volvimento de $f\left(x_{j} ; B\right)$ em série de Taylor, sujeita-se a diver gir, Levenberg adota para ser minimizada uma soma de quadrados ponderada,

$$
\bar{S}(B)=v S(B)+\sum_{k=1}^{m} w_{k}\left(b_{k}-b_{k i}\right)^{2}
$$

$\operatorname{com} \underline{v}$ e $w_{k}$ constantes positivas que representam a importância 
relativa de $\mathrm{S}(\mathrm{B})$ e de $\left(\mathrm{b}_{\mathrm{k}}-\mathrm{b}_{\mathrm{ki}}\right)$ no processo de minimização. Aplicando o método dos quadrados inínimos, obtém a fórmula ite rativa:

$$
B_{i+1}=B_{i}-L_{i}^{-1} G_{i}
$$

com $L_{i}$ uma matriz (mxm) que difere da matriz do método de Gauss, $\tilde{H}_{i}$, apenas pelos elementos da diagonal principal, os quais, na matriz $L_{i}$, estão acrescidos de valores $w_{k} v^{-1}$ escolhidos de modo a assegurar a convergência. Além disso, os dois métodos coincidem quando $v \rightarrow \infty$. A utilidade prática deste método depende das constantes $\underline{v}$ e $w_{k}$ serem selecionadas por critérios que levem a procedimentos adequados para sua progra mação em computadores.

HARTLEY (1961) introduz uma modificação no mé todo de Gauss considerando, em cada iteração, a função

$$
S(p)=S\left(B_{i}-p \tilde{H}_{i}^{-1} G_{i}\right) \quad, \quad 0 \leq p \leq 1
$$

Atribuindo três valores para $\underline{p}$ (por exemplo:0,1/2 e 1), obtém três pontos, e determinando o mínimo $\mathrm{p}^{*}$ da parábola que passa por eles adota a fórmula

$$
\mathrm{B}_{i+1}=\mathrm{B}_{\mathbf{i}}-\mathrm{p}^{*} \tilde{\mathrm{H}}_{\mathbf{i}}^{-1} \mathrm{G}_{\mathbf{i}}
$$

Considera, também, que haverá convergência sempre que o ’ processo se inicie numa região convexa em torno do valor a ser alcançado .

FLETCHER e POWELL (1963) apresentam um método 
iterativo de grande poder, baseado num procedimento descrito por DAVIDON (1959), no qual a matriz Ré sistematicamente ajus tada de iteração em iteração, pretendendo-se um comportamento semelhante ao da inversa da Hessiana. Alguns teoremas sobre a convergência são provados e se efetuam vários testes pa ra exemplificá-los. Implementações alternativas a esse método fo ram introduzidas por BARD (1970) e também por FLETCHER (1970). MARQUARDT (1963), com bases teóricas mais sólí das, chega numa fórmula iterativa semelhante à de Levenberg, com

$$
\mathrm{w}_{\mathrm{k}}=\sum_{j=1}^{\mathrm{n}}\left[\left(\partial \mathrm{f}\left(\mathrm{x}_{\mathrm{j}} ; \mathrm{B}\right) / \partial \mathrm{b}_{\mathrm{k}}\right)^{2}\right]_{\mathrm{B}=\mathrm{B}_{i}}
$$

e $\mathrm{v}^{-1}=\mathrm{q}$ escolhido convenientemente. Propõe, também, um algoritmo para a aplicação do processo iterativo. Este método tem se mostrado de grande eficiência, principalmente por, em geral, permitir a adoção de $\underline{q}$ tal que tenhamos $S\left(B_{i+1}\right)<S\left(B_{i}\right)$. KITTRELL et alii. (1965), destacando que a con vergência dos processos iterativos depende das estimativas iniciais dos parâmetros e da complexidade da equação, fornecem algumas técnicas para a obtenção dessas estimativas. Uma primeira sugere usar as informações disponíveis a respeito da magnitú de esperada para os parâmetros desconhecidos. Estas podem ser intervalos ou estimativas mais definidas dos valores dos parâmetros. Outra técnica é a estimação cíclica, pela qual, uma parte dos parâmetros é mantida fixa enquanto a outra varia. Uma terceira recomenda linearizar a equação do modelo, se for 
possível, e pelo método dos quadrados mínimos determinar as estimativas iniciais. Observemos que, muitas vezes, os pesqui sadores afirmam terem feito uma linearização quando, na verdade, mudaram o modelo. Assim, supondo originalmente

$$
\mathrm{Y}=\mathrm{f}(\mathrm{X})+\mathrm{E}
$$

a aplicação de logarítmos daria

$$
\log \mathrm{Y}=\log [\mathrm{f}(\mathrm{X})+\mathrm{E}],
$$

mas, como se adota

$$
\log Y=\log f(X)+E
$$

não podemos dizer que o modelo inicial foi linearizado. Os $\underline{a} \underline{\underline{u}}$ tores discutem, ainda, a pesquisa em "rede", dividindo a superfície de somas de quadrados em partes, com o mínimo procu rado em cada uma delas. Exemplificam com problemas ligados à Química e chamam a atenção de que a escolha de uma técnica particular depende do problema em questão.

SMITH e SHANNO (1971) descrevem um procedimen to para resolver problemas de estimação não linear, resumindo se numa modificação do algoritmo de Marquardt, de modo que $\underline{q}$, antes com a restrição de não ser negativo, assuma certos valo res negativos quando vantajoso. Entretanto, por incluir o cál culo de autovalores, o método tem sido considerado trabalhoso. Quanto às considerações probabilísticas, após a determinação das estimativas dos parâmetros, a literatura é 
um tanto limitada. Além de BARD (1974) já citado, destaca-se o artigo de HARTLEY (1964) que trata de regiões de confiança para os parâmetros em leis de regressão não linear. GUTTMAN e MEETER (1965), através de exemplos numéricos, examinam a valí dade das medidas de não linearidade. Também é importante o tex to de DRAPER e SMITH (1981).

Cabe, agora, uma revisão de literatura para as Leis de Mitscherlich com ênfase à Segundalei quando aplicada a dados de maturação da cana-de-açúcar. A Primeira Lei,

$$
Y=b_{1}\left[1-10^{-b_{3}\left(X+b_{2}\right)}\right]
$$

divulgada na Alemanha, em 1909, é aceita atualmente como apro priada ao estudo de ensaios de adubação de plantas cultivadas, com X representando a dose de um certo nutriente e Y a produção correspondente. Seu comportamento pode ser visualizado pelo gráfico:

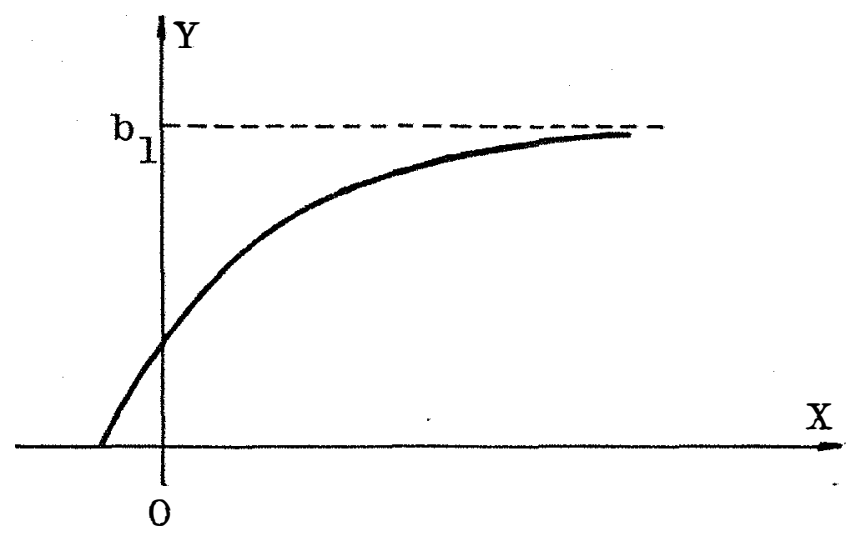

Muitos trabalhos foram apresentados sobre ela destacando-se os de PIMENTEL GOMES $(1951,1953,1969,1978)$ e alguns deste mes mo autor em colaboração com outros $(1949,1966,1967,1975)$. 
Tais estudos resultaram num processo geral para estimar os pa râmetros da Primeira Lei; em considerações sobre sua aplicabilidade na análise de experimentos de adubação e na comparação, e indicação de dificuldádes mais comuns, quando se em pregam polinômios com o mesmo objetivo.

Em 1928, Mitscherlich propôs uma modificação na sua equação, acrescentando o fator

$$
10^{-b_{4}\left(x+b_{2}\right)^{2}}
$$

que possibilita um decrescimento na curva a partir de um cer to valor de $\mathrm{X}$, quando $\mathrm{b}_{4}$ é positivo, criando, assim, a sua conhecida Segunda Lei. Esta é cogitada em trabalhos sobre a Primeira, mas sua utilização é preterida devido aos cálculos que requer e por se considerarem satisfatórios, nessas pesquisas, os resultados da Primeira Lei.. Seu uso é recomendado quando doses excessivas do nutriente são empregadas.

SILVA (1978) estuda a adequação da Segunda Lei de Mitscherlich a dados de adubação de trigo, e compara os re sultados obtidos com os provenientes do ajustamento da Primeira Lei e do modelo. Quadrático aos mesmos dados. obtém estimativas dos parâmetros do modelo de Mitscherlich através do método de Gauss, com a modificação de Hartley, atingindo inclusive uma solução com $b_{1}$ e $b_{3}$ negativos, e do modelo Quadrá tico pelo método usual de quadrados mínimos para modelos lineares. Conclui que a Segunda Lei de Mitscherlich se adapta bem aos dados, sendo sua aplicação preferível quando se aplí 
cam altas doses de fertilizante, caso em que há uma queda na produção a partir de certo nível.

SILVA (1980) adota $b_{2}=0$ na Segunda Lei de Mits cherlich e estuda seu ajustamento a dados referentes à maturação da cana-de-açúcar. Emprega, para a estimação dos parâmetros, os métodos de Newton, Gauss (modificado) e Marquardt com o objetivo de verificar qual o melhor para o modelo em questão. Conclui que o método de Marquardt chega à solução em um número menor de iterações, ainda que os outros também levem à boas estimativas. Entretanto, a ausência do fator $b_{2}$ tornou, pelo modelo, nula a quantidade de sacarose na cana no mês anterior ao do início das medições, o que não ocorre na prática.

UDO (1983) aplica os modelos: Segunda Lei de Mitscherlich, Quadrático e da Raiz Quadrada,.também para veri ficar qual deles é mais apropriado ao estudo de maturação da cana-de-açúcar. Para a estimação dos parâmetros da Lei de Mitscherlich utiliza o método de Marquardt. Deduz, aplicando logaritmo na função de Mitscherlich, que os parâmetros $b_{1} e$ $b_{3}$ devem ser positivos, porém, esta conclusão não é válida, por ter sido obtida da restrição de só existir, no campo real, logaritmo de número positivo. Adotando os testes estatísticos para modelos lineares, conclui que o Quadrático se adapta me lhor ao fenômeno do que a Lei de Mitscherlich, porém que esta última é mais adequada à extrapolação. 
3. MATERIAL E MÉTODOS

3.1. Material

Este trabalho adota, para estudar o ajuste do modelo Quadrático e da Segunda Lei de Mitscherlich ao fenôme no de maturação da cana-de-açúcar, resultados analíticos de Pol\% da cana referentes a ensaios de competição de variedades realizados na região de Sertãozinho (S.P.), no ano de 1970, e na Usina São João, Araras (S.P.), no ano de 1979. Esses dạ dos foram utilizados por SILVA (1980) e UDO (1983) respectivamente, e estão expostos, aqui, na Tabela 1 (pag. 43 ) onde as variedades de 1 a 19 são as da primeira autora e as de 20 a 27 as da segunda. Os dados destas oito últimas variedades são médias de três repetições, as quais estão apresentadas na Tabela 7 do apêndice.

De acordo com BAYMA (1975), a "Pol", medida for 
necida pelos aparelhos chamados Polarímetros e pelos Sacaríme tros, é uma soma algébrica do poder xotatóriodos açúcares da cana sobre a luz polarizada. Na prática, se a cana está madura e sadia, a porcentagem de açúcares redutores é baixa e a medição de Pol estima o teor de sacarose.

3.2. Método dos Quadrados Mínimos

3.2.1. Modelo da Regressão Quadrática

Consideremos que $\underline{n}$ valores $Y_{j}$ de Pol\% dacana, obtidos em $\underline{n}$ épocas $x_{j}$, relacionam-se pelo modelo

$$
\mathrm{Y}_{j}=\mathrm{b}_{0}+\mathrm{b}_{1} \mathrm{X}_{j}+\mathrm{b}_{2} \mathrm{X}_{j}^{2}+\mathrm{E}_{j} \quad(j=1,2, \ldots, \mathrm{n} ; \mathrm{n}>3)
$$

onde os erros $E_{j}$ são variáveis aleatórias independentes de mé dia zero e variância constante $\sigma^{2}$. Admitimos, ainda, que os erros tenham distribuição normal.

Em notação matricial o modelo fica

$$
\mathrm{Y}=\mathrm{XB}+\mathrm{E}
$$

sendo

$$
\mathrm{Y}=\left[\begin{array}{c}
\mathrm{Y}_{1} \\
\mathrm{Y}_{2} \\
\vdots \\
\mathrm{Y}_{\mathrm{n}}
\end{array}\right], \quad \mathrm{X}=\left[\begin{array}{ccc}
1 & \mathrm{X}_{1} & \mathrm{X}_{1}^{2} \\
1 & \mathrm{X}_{2} & \mathrm{X}_{2}^{2} \\
\vdots & \vdots & \vdots \\
1 & \mathrm{X}_{\mathrm{n}} & \mathrm{X}_{\mathrm{n}}^{2}
\end{array}\right], \quad \mathrm{B}=\left[\begin{array}{c}
\mathrm{b}_{0} \\
\mathrm{~b}_{1} \\
\mathrm{~b}_{2}
\end{array}\right], \quad \mathrm{E}=\left[\begin{array}{c}
\mathrm{E}_{1} \\
\mathrm{E}_{2} \\
\vdots \\
\mathrm{E}_{\mathrm{n}}
\end{array}\right]
$$


$\operatorname{com} \operatorname{Esp}(E)=\Phi, \quad \operatorname{Esp}\left(E^{\prime}\right)=I \sigma^{2} \quad(I=M a t r i z$ de Identidade $)$

\subsubsection{Estimação dos Parâmetros}

A estimativa de B pel o método dos quadrados mínimos é o vetor $\hat{\mathrm{B}}=\left[\hat{\mathrm{b}}_{0}, \hat{\mathrm{b}}_{1}, \hat{\mathrm{b}}_{2}\right]^{\prime}$, o qual minimiza a soma de quadrados dos erros E'E. Considerando identicamente nula a diferencial des ta soma em relação aos parâmetros, chegamos ao sistema de equações normais

$$
X^{\prime} X \hat{B}=X^{\prime} Y
$$

que, para X'X não-singular, fornece a solução

$$
\hat{\mathrm{B}}=\left(\mathrm{X}^{\prime} \mathrm{X}\right)^{-1} \mathrm{X} \cdot \mathrm{Y}
$$

isto é,

$$
\left[\begin{array}{c}
\hat{b}_{0} \\
\hat{b}_{1} \\
\hat{b}_{2}
\end{array}\right]=\left[\begin{array}{ccc}
n & \sum_{j=1}^{n} x_{j} & \sum_{j=1}^{n} x_{j}^{2} \\
\sum_{j=1}^{n} x_{j} & \sum_{j=1}^{n} x_{j}^{2} & \sum_{j=1}^{n} x_{j}^{3} \\
\sum_{j=1}^{n} x_{j}^{2} & \sum_{j=1}^{n} x_{j}^{3} & \sum_{j=1}^{n} x_{j}^{4}
\end{array}\right] \cdot\left[\begin{array}{c}
\sum_{j=1}^{n} Y_{j} \\
\sum_{j=1}^{n} Y_{j} x_{j} \\
\sum_{j=1}^{n} Y_{j} x_{j}^{2}
\end{array}\right] .
$$

Determinadas as estimativas dos parâmetros, en tão, estimativas $\hat{Y}$ de Y podem ser obtidas pela equação ajus- 
18.

tada: $\quad \hat{Y}=\hat{b}_{0}+\hat{b}_{1} X+\hat{b}_{2} X^{2}$.

3.2.3. Análise de Variância

Após a determinação da equação de regressão, podemos conduzir a análise da variância, conforme o esquema seguinte :

\begin{tabular}{|c|c|c|c|}
\hline Causa de Variação & G.L. & $S \cdot Q$. & $Q \cdot M$. \\
\hline Regressão & $m-1=2$ & $\hat{B}^{\prime} X^{\prime} Y-C$ & $\left(\hat{B}^{\prime} X^{\prime} Y-\mathbb{C}\right) / 2$ \\
\hline $\begin{array}{c}\text { Desvios da Regressão } \\
\text { (Resíduo) }\end{array}$ & $n-3$ & $Y^{\prime} Y-\hat{B}^{\prime} X^{\prime} Y$ & $\left(Y^{\prime} Y-\hat{B}^{\prime} X^{\prime} Y\right) /(n-3)$ \\
\hline Total & $n-1$ & $Y^{\prime} Y-C$ & \\
\hline
\end{tabular}

Um outro procedimento para a obtenção da soma de quadrados dos desvios da regressão seria:

$$
\text { SQ Desvios }=\sum_{j=1}^{n}\left(Y_{j}-\hat{Y}_{j}\right)^{2} .
$$

Para testar a hipótese $\mathrm{H}_{0}: \mathrm{b}_{1}=\mathrm{b}_{2}=0$, ao nível de significância $\alpha$, podemos utilizar a estatística F. Nesse caso, rejeitamos $H_{0}$ sempre que o valor de

$$
F=\frac{Q M \text { Regressão }}{\text { QM Desvios }}
$$


for maior do que o valor crítico $F(2, n-3, d)$.

A proporção da variação total de Y "explicada" pela regressão é medida pelo Coeficiente de Determinação

$$
\mathrm{R}^{2}=\frac{\mathrm{SQ} \text { Regressão }}{\text { SQ Total }}
$$

obtido de $S Q$ Total = SQRegressão + SQ Desvios e, portanto, quan to mais próximo de 1 estiver $\mathrm{R}^{2}$ melhor será o ajustamento, pois, $0 \leq R^{2} \leq 1$. Este coeficiente tende a crescer quando $\underline{\mathrm{n}} \underline{\mathrm{di}}$. minui e quando aumenta o grau do polinômio e, acompanhando HOFFMANN e VIEIRA (1977), deve ser corrigido para o número de graus de liberdade obtendo-se o Coeficiente de Determinação Corrigido:

$$
\begin{aligned}
\overline{\mathrm{R}}^{2} & =1-\frac{\text { SQ Desvios } /(n-m)}{\text { SQ Total/(n-1) }} \\
& =\mathrm{R}^{2}-\frac{\mathrm{m}-1}{\mathrm{n}-\mathrm{m}}\left(1-\mathrm{R}^{2}\right)
\end{aligned}
$$

Um outro indicador da qualidade do ajustamento obtido é o Coeficiente de Variação, definido por

$$
\mathrm{C} \cdot \mathrm{V} \cdot=\frac{\sqrt{\mathrm{QM} \text { Desvios }}}{\overline{\mathrm{Y}}}
$$

que mede a dispersão relativa das observações. O resultado se rá tanto melhor quanto menor for o Coeficiente de Variação.

$\mathrm{Na}$ análise anterior, adotamos como estimativa da variância do erro experimental o quadrado médio dos desvios da regressão. Quando dispomos, para um ou mais valores de $X$, de mais de um valor observado de $Y$, é possível obter 
uma estimativa melhor da variância do erro, dada pelo quadrado médio do resíduo de una análise de variância em que cada valor distinto de $\mathrm{X}$ é suposto como um "tratamento" a que es tá sendo submetida a variável $\mathrm{Y}$. Considerando $\mathrm{Y}_{j \mathrm{k}}$ a observação $\underline{\mathrm{k}}$ da época $\underline{\mathrm{j}} \quad(\mathrm{j}=1,2, \ldots, \mathrm{n}$ e $\mathrm{k}=1,2, \ldots, \mathrm{r})$, teremos as somas de quadrados:

SQ Total $=\sum_{k=1}^{r} \sum_{j=1}^{n} Y_{j k}^{2}-C \quad$ onde $\quad C=\left(\sum_{k=1}^{r} \sum_{j=1}^{n} Y_{j k}\right)^{2} / n r$,
$S Q$ Tratamentos $=\frac{1}{r} \sum_{j=1}^{n} T_{j}^{2}-C \quad$ com $T_{j}=$ Total do tratam. $j, e$ SQ Resíduo = SQ Total - SQ Tratamentos .

Podemos decompor a SQ Tratamentos para estudar a regressão e, levando em conta os objetivos deste trabalho, indicamos:

SQ Tratamentos = SQ Regressão + SQ Desvios da regressão onde $S Q$ Desvios da regressão $=r \sum_{j=1}^{n}\left(\bar{Y}_{j}-\hat{Y}_{j}\right)^{2}$

com $\overline{\mathrm{Y}}_{j}$ representando a média das observações da época $\mathrm{x}_{\mathrm{j}}$. o esquema da análise de variância será:

\begin{tabular}{ll}
\hline Causa de variação & G.L. \\
\hline Regressão & $\mathrm{m}-1=2$ \\
Desvios da Regressão & $\mathrm{n}-\mathrm{m}$ \\
\hline (Tratamentos) & $(\mathrm{n}-1)$ \\
Resíduo & $\mathrm{n}(\mathrm{r}-1)$ \\
\hline Total & $\mathrm{nr}-1$ \\
\hline
\end{tabular}


Teremos :

$$
F_{\text {regr }}=\frac{Q M \text { Regressão }}{\text { QMesíduo }}
$$

e

$$
\mathrm{F}_{\text {desv } \cdot \text { regr }}=\frac{\mathrm{QM} \text { Desvios da regr }}{\mathrm{QM} \text { Resíduo }}
$$

e uma conclusão de que os dados apresentem a suposta relação quadrática entre as variáveis $\mathrm{X}$ e $\mathrm{Y}$, através de um valor significativo de Fregr, deve vir acompanhada de um valor não significativo de F desv.regr.

\subsubsection{Programação do Método}

Desenvolvemos, aqui, um programa em linguagem BASIC voltado especificamente para a regressão Quadrática. De finindo as somas de produtos, para $\underline{n} \operatorname{pares}\left(\mathrm{Z}_{j}, \mathrm{~W}_{\mathbf{j}}\right)$,

$$
S(z, w)=\sum_{j=1}^{n}\left(Z_{j}-\bar{Z}\right)\left(w_{j}-\bar{W}\right)=\sum_{j=1}^{n} Z_{j} W_{j}-\left(\sum_{j=1}^{n} Z_{j}\right)\left(\sum_{j=1}^{n} W_{j}\right) / n
$$

não é difícil, a partir do sistema de equações normais, chegar às seguintes fórmulas: 


$$
\begin{aligned}
& \hat{b}_{1}=\frac{S(x, y) S\left(x^{2}, x^{2}\right)-S\left(x^{2}, y\right) S\left(x, x^{2}\right)}{S(x, x) S\left(x^{2}, x^{2}\right)-\left(S\left(x, x^{2}\right)\right)^{2}} \\
& \hat{b}_{2}=\frac{S(x, x) S\left(y, x^{2}\right)-S\left(x, x^{2}\right) S(x, y)}{S(x, x) S\left(x^{2}, x^{2}\right)-\left(S\left(x, x^{2}\right)\right)^{2}} \\
& \hat{b}_{0}=\bar{Y}-\hat{b}_{1} \bar{x}-\hat{b}_{2} \bar{x}^{2}
\end{aligned}
$$

A programação se baseia na formação de uma mạ triz $X$ com elementos $X(1, j)=X_{j}, X(2, j)=X_{j}^{2}$ e $X(3, j)=Y_{j}$ para $j=1,2, \ldots, n$, possibilitando chegarmos às somas $S$ pela com binação dos índices 1,2 e 3, dois a dois. Observemos que o programa foi elaborado para dados sem repetição e, quando es tas existirem, os parâmetros serão estimados corretamente des de que $\mathrm{Y}_{j}$ represente a média das observações da época $\mathrm{X}_{j}$, mas, para a análise estatística, deverá sofrer as modificações in dicadas no final da seção anterior.

5 REM: REGRESSAO QUADRATICA

$1 \emptyset$ PRINT "QUAL O NO. DE OBSERVACOES",

$2 \varnothing$ INPUT $\mathrm{N}$

$3 \varnothing \operatorname{DIM} \mathrm{R}(3), \mathrm{S}(3,3), \mathrm{T}(3,3), \mathrm{X}(3, \mathrm{~N})$

37 REM: ENTRAM AS OBSERVACOES

39 REM: CALCULA SOMAS DE $\mathrm{X}, \mathrm{DE} \mathrm{X} * \mathrm{X}$ E DE $\mathrm{Y}$

$4 \varnothing$ PRINT "ENTRE OS PARES $\mathrm{X}, \mathrm{Y}$ "

$5 \varnothing$ FOR $\mathrm{J}=1$ TO $\mathrm{N}$.

$6 \varnothing$ PRINT $J$,

$7 \varnothing \operatorname{INPUT} \mathrm{X}(1, \mathrm{~J}), \mathrm{X}(3, \mathrm{~J})$

$8 \varnothing \mathrm{X}(2, \mathrm{~J})=\mathrm{X}(1, \mathrm{~J}) * \mathrm{X}(1, \mathrm{~J})$

$9 \varnothing \mathrm{R}(1)=\mathrm{R}(1)+\mathrm{X}(1, \mathrm{~J})$

$1 \varnothing \varnothing \mathrm{R}(2)=\mathrm{R}(2)+\mathrm{X}(2, \mathrm{~J})$

$11 \varnothing \mathrm{R}(3)=\mathrm{R}(3)+\mathrm{X}(3, \mathrm{~J})$ 


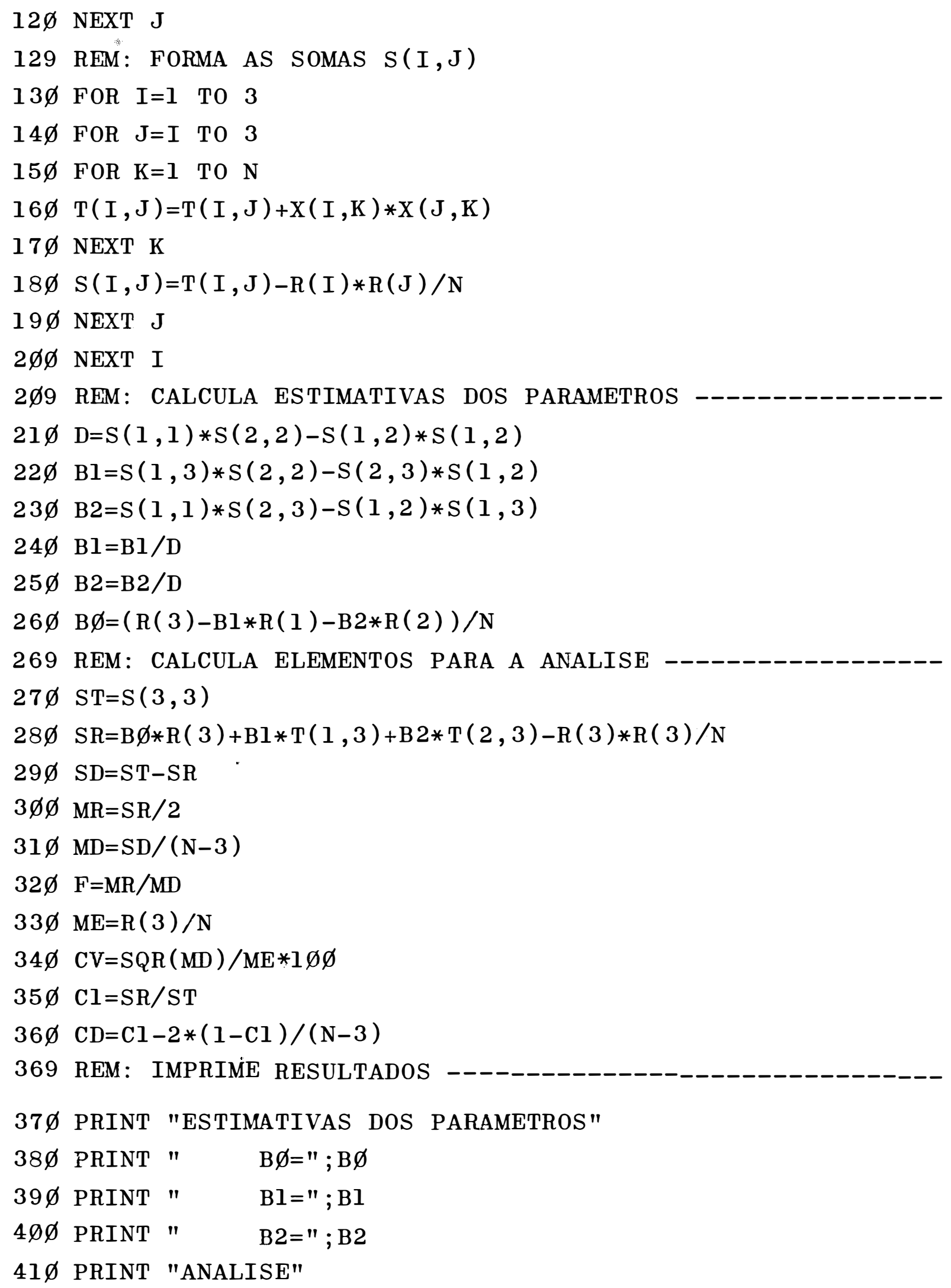


$42 \varnothing$ PRINT " SQDESV=";SD

$43 \emptyset$ PRINT " QMDESV $=" ; \mathrm{MD}$

$44 \varnothing$ PRINT " $\mathrm{F}=" ; \mathrm{F} ; "(2 \mathrm{E} " ; \mathrm{N}-3 ;$ "GRAUS DE LIBERDADE $) "$

$45 \varnothing$ PRINT " C.DET. $=" ; \mathrm{CD}$

$46 \varnothing$ PRINT " C.VAR. $=" ; \mathrm{CV}$

$47 \varnothing$ END

Quando tivermos $\hat{b}_{2}<0$ haverá um valor máximo de Pol\% da cana determinado com a introdução das seguintes linhas no programa:

462 IF B2>Ø THEN 468

$463 \mathrm{XM}=-\mathrm{B} 1 /(2 * \mathrm{~B} 2)$

$464 \mathrm{YM}=\mathrm{B} \varnothing-\mathrm{Bl} * \mathrm{Bl} /(4 * \mathrm{~B} 2)$

465 PRINT

466 PRINT "XMAX="; XM;" YMAX=";YM

467 GOTO $47 \varnothing$

468 PRINT "NAO TEM MAXIMO"

3.3. Métodos do Gradiente

3.3.1. Modelo da Segunda Lei de Mitscherlich

Consideremos, agora, que os $\underline{n}$ valores $Y_{j}$ de Pol\% da cana, obtidos para as $\underline{\mathrm{n}}$ épocas $\mathrm{x}_{\mathrm{j}}$, sigam o modelo

$$
\mathrm{Y}_{j}=\mathrm{f}\left(\mathrm{X}_{\mathrm{j}} ; \mathrm{B}\right)+\mathrm{E}_{\mathrm{j}} \quad(\mathrm{j}=1,2, \ldots, \mathrm{n} ; \mathrm{n}>4)
$$

onde

$$
f\left(x_{j} ; B\right)=b_{1}\left[1-10^{-b_{3}\left(x_{j}+b_{2}\right)}\right] 10^{-b_{4}\left(x_{j}+b_{2}\right)^{2}}
$$


e os erros $E_{j}$ são variáveis aleatórias independentes com dis tribuição normal de inédia zero e variância $\sigma^{2}$. Como anterior mente, na impossibilidade de conhecermos o vetor correto de parâmetros, $\quad B=\left[b_{1}, b_{2}, b_{3}, b_{4}\right]$, , adotamos uma estimativa $\hat{\mathrm{B}}=\left[\hat{\mathrm{b}}_{1}, \hat{\mathrm{b}}_{2}, \hat{\mathrm{b}}_{3}, \hat{\mathrm{b}}_{4}\right]^{\prime}$ que minimize a soma de quadrados dos erros

$$
S(B)=\sum_{j=1}^{n}\left[x_{j}-f\left(x_{j} ; B\right)\right]^{2} .
$$

Uma condição necessária e suficiente para que $\hat{\mathrm{B}}$ seja um ponto de mínimo de $\mathrm{S}(\mathrm{B})$ é que, neste ponto, se anu le o vetor gradiente $G(B)$ de $S$ e seja definida positiva a matriz Hessiana $\mathrm{H}(\hat{\mathrm{B}})$.

Adotando as notações:

$$
\begin{aligned}
& \mathrm{f}_{\mathrm{j}}=\mathrm{f}\left(\mathrm{X}_{\mathrm{j}} ; \mathrm{B}\right) \\
& (j=1,2, \ldots, n) \\
& f_{k j}=\partial f\left(x_{j} ; B\right) / \partial b_{k} \text {, } \\
& (\mathrm{k}, \mathrm{m}=1,2,3,4) \\
& f_{k m j}=\partial^{2} f\left(x_{j} ; B\right) / \partial b_{k} \partial b_{m},
\end{aligned}
$$

e considerando as variáveis auxiliares:

$$
\begin{aligned}
& \mathrm{z}_{\mathrm{j}}=\mathrm{x}_{\mathrm{j}}+\mathrm{b}_{2} \\
& U_{j}=10^{-b_{3}\left(x_{j}+b_{2}\right)}=10^{-b_{3} z_{j}} \text {, } \\
& \mathrm{V}_{\mathbf{j}}=\mathrm{I}-\mathrm{U}_{\mathbf{j}} \\
& w_{j}=10^{-b_{4}\left(x_{j}+b_{2}\right)^{2}}=10^{-b_{4} z_{j}^{2}} \text {, }
\end{aligned}
$$

teremos para a Segunda Lei de Mitscherlich a equação 


$$
\mathrm{f}_{j}=\mathrm{b}_{1} \mathrm{~V}_{\mathrm{j}} \mathrm{w}_{\mathrm{j}}
$$

As derivadas parciais de primeira ordem serão:

$$
\begin{aligned}
& f_{1 j}=v_{j} W_{j}, \\
& f_{2 j}=b_{1}(L 10) w_{j}\left(b_{3} U_{j}-2 b_{4} Z_{j} V_{j}\right), \quad \text { onde } L 10=\log _{e} 10, \\
& f_{3 j}=b_{1}(L 10) z_{j} U_{j} W_{j}, \\
& f_{4 j}=-b_{1}(L 10) z_{j}^{2} v_{j} W_{j},
\end{aligned}
$$

e as derivadas parciais de segunda ordem serão:

$$
\begin{aligned}
& f_{11 j}=0, \\
& f_{12 j}=(L 10) w_{j}\left(b_{3} U_{j}-2 b_{4} Z_{j} V_{j}\right), \\
& \mathrm{f}_{13 \mathrm{j}}=(\mathrm{Ll} 0) \mathrm{Z}_{j} \mathrm{U}_{\mathbf{j}} \mathrm{w}_{\mathbf{j}}, \\
& f_{14 j}=-(L 10) z_{j}^{2} V_{j} W_{j} \\
& f_{22 j}=-b_{1}(L 10) w_{j}\left\{b_{3}(L 10) U_{j}\left(b_{3}+4 b_{4} z_{j}\right)+2 b_{4} V_{j}\left[1-2 b_{4}(L 10) z_{j}^{2}\right]\right\} \text {, } \\
& f_{23 j}=b_{1}(L 10) U_{j} W_{j}\left[1-(L 10) z_{j}\left(b_{3}+2 b_{4} z_{j}\right)\right], \\
& f_{24 j}=-b_{1}(L 10) z_{j} W_{j}\left\{2 V_{j}\left[1-b_{4}(L l 0) z_{j}^{2}\right]+b_{3}(L 10) z_{j} U_{j}\right\} \text {, } \\
& f_{33 j}=-b_{1}(L 10)^{2} z_{j}^{2} U_{j} w_{j} \\
& \mathrm{f}_{34 \mathrm{j}}=-\mathrm{b}_{1}(\mathrm{Ll} l 0)^{2} \mathrm{z}_{j}^{3} \mathrm{U}_{j} \mathrm{~W}_{j} \text {, } \\
& f_{44 j}=b_{1}(L 10)^{2} z_{j}^{4} v_{j} w_{j} \text {, }
\end{aligned}
$$




$$
\begin{aligned}
& f_{21 j}=f_{12 j}, f_{31 j}=f_{13 j}, f_{41 j}=f_{14 j}, \\
& f_{32 j}=f_{23 j}, \quad f_{42 j}=f_{24 j}, f_{43 j}=f_{34 j} .
\end{aligned}
$$

Resulta para S(B) um vetor gradiente G de com ponentes $g_{k} \quad(k=1,2,3,4)$,

$$
g_{k}=\partial S / \partial b_{k}=-2 \sum_{j=1}^{n}\left(Y_{j}-f_{j}\right) f_{k j}
$$

e uma matriz Hessiana de elementos $h_{k m}(k, m=1,2,3,4)$,

$$
\mathrm{h}_{\mathrm{km}}=\partial^{2} \mathrm{~S} / \partial \mathrm{b}_{\mathrm{k}} \partial \mathrm{b}_{\mathrm{m}}=2 \sum_{j=1}^{\mathrm{n}} \mathrm{f}_{\mathrm{kj}} \mathrm{f}_{\mathrm{mj}}-2 \sum_{j=1}^{\mathrm{n}}\left(\mathrm{Y}_{j}-\mathrm{f}_{j}\right) \mathrm{f}_{\mathrm{kmj}} \text {. }
$$

Observamos, por esses resultados, que a deter minação algébrica de um vetor $\hat{B}$ que anule o gradienteG, pela resolução do sistema $G=0$, é impraticável. Por isso, métodos especiais devem ser empregados.

\subsubsection{Método de Newton}

Em lugar de S(B), trabalhamos com uma aproximação como descrito a seguir. Tomando $H_{i}$ e $G_{i}$, respectivamen te a matriz Hessiana e o vetor gradiente de $S$ calculados para um vetor $B_{i}=\left[b_{1 i}, b_{2 i}, b_{3 i}, b_{4 i}\right]$ ', definimos a função

$$
\mathrm{s}_{\mathbf{i}}(\mathrm{B})=\mathrm{S}\left(\mathrm{B}_{\mathbf{i}}\right)+\mathrm{G}_{\mathbf{i}}^{\prime}\left(\mathrm{B}-\mathrm{B}_{\mathbf{i}}\right)+\frac{1}{2}\left(\mathrm{~B}^{\left.-\mathrm{B}_{\mathbf{i}}\right)} \cdot \mathrm{H}_{\mathbf{i}}\left(\mathrm{B}-\mathrm{B}_{\mathbf{i}}\right)\right.
$$

que consiste nos termos até segunda ordem do desenvolvimento em série de Taylor de $\mathrm{S}(\mathrm{B})$, em torno de $\mathrm{B}_{\dot{i}}$.Considerando iden 
ticamente nula a diferencial de $s_{i}(B)$ em relação ao $B$, obtemos

$$
\mathrm{G}_{\mathbf{i}}+\mathrm{H}_{\mathbf{i}}\left(\mathrm{B}-\mathrm{B}_{\mathbf{i}}\right)=0
$$

que, se $\mathrm{H}_{i}$ for não-singular, fornece o vetor $\mathrm{B}=\mathrm{B}_{i+1}$ para 0 qual é nulo o gradiente de $\mathrm{s}_{i}(\mathrm{~B})$ :

$$
\mathrm{B}_{\mathbf{i}+1}=\mathrm{B}_{\mathbf{i}}-\mathrm{H}_{\mathbf{i}}^{-1} \mathrm{G}_{\mathbf{i}}
$$

Esta fórmula leva diretamente ao mínimo quando $\mathrm{S}(\mathrm{B})$ é uma função quadrática e $\mathrm{H}_{i}$ é uma matriz definida posi tiva, conforme a condição necessária e suficiente da seção an terior. Entretanto, $B_{i+1}$ dificilmente coincidirá com o mínimo quando $S(B)$ não é quadrática e, neste caso, a fórmula define a i-ésima iteração do método de Newton. Assim, o método consiste em calcular correções sucessivas para uma solução inicial $B_{1}$ até que a correção $B_{i+1}-B_{i}$, que temo gradiente, seja considerada desprezível. Admitimos que está havendo convergência enquanto permanecer $S\left(B_{i+1}\right)<S\left(B_{i}\right)$. Isso deve ocorrer para $\mathrm{H}_{i}^{-1}$ definida positiva, pois a derivada de $\mathrm{S}$ na direção do vetor correção, $-G_{i} H_{i}^{-1} G_{i}$, será negativa.

$\mathrm{Na}$ prática, como nem sempre teremos a anulação do gradiente, a correção pode ser considerada desprezível quando o valor absoluto dos componentes de $B_{i+1}-B_{i}$ for menor do que uma precisão pré-formulada. BARD (1974) sugere que o procedimento seja interrompido quando

$$
\left|b_{k(i+1)}-b_{k i}\right|<e_{i} \quad(k=1,2,3,4)
$$


com

$$
\mathrm{e}_{\mathrm{i}}=10^{-4}\left(\mathrm{~b}_{\mathrm{ki}}+10^{-3}\right)
$$

sendo $10^{-3}$ colocado para evitar dificuldades nas proximidades de zero.

\subsubsection{Método de Gauss}

Quando da revisão de literatura, o método de Gauss foi apresentado como uma sequência de resoluções de prọ blemas lineares, substituindo a equação não linear do modelo por uma aproximação linear. 0 método pode ser visto, ainda, como uma aproximação do método de Newton, considerando a matriz $\tilde{H}$, em lugar de H, obtida da eliminação nesta última, em cada elemento, do termo que contém as derivadas parciais de segunda ordem, ou seja, $\tilde{H}$ é formada pelos elementos

$$
\tilde{h}_{\mathrm{km}}=2 \sum_{j=1}^{\mathrm{n}} \mathrm{f}_{\mathrm{kj}} \mathrm{f}_{\mathrm{mj}} \quad(\mathrm{k}, \mathrm{m}=1,2,3,4)
$$

os termos abandonados contêm os erros $\mathrm{Y}_{j}-\mathrm{f}_{\mathbf{j}} \mathrm{e}$ se estes forem pequenos, o que deve acontecer pelo menos nas proximidades do mínimo, ்̃ será uma boa aproximação de H. Então, no método de Gauss as iterações são definidas por:

$$
\mathrm{B}_{i+1}=\mathrm{B}_{i}-\tilde{\mathrm{H}}_{\mathbf{i}}^{-1} \mathrm{G}_{\mathbf{i}}
$$

A condição de que esta fórmula, em cada iteração, produza uma 
soma de quadrados menor do que a anterior pode falhar, princi palmente quando $\tilde{H}_{i}$ nãu é uma boa aproximação de $H_{i}$, implicando numa correção grande. Este problema é contornado considerando

$$
\mathrm{B}_{\mathbf{i}+1}=\mathrm{B}_{\mathbf{i}}-\mathbf{p} \tilde{\mathrm{H}}_{\mathbf{i}}^{-1} \mathrm{G}_{\mathbf{i}}
$$

de modo que $\underline{\mathrm{p}}$ suficientemente pequeno forneça $S\left(B_{i+1}\right)<S\left(B_{i}\right)$. HARTLEY (1961) propõe que p seja determinado de forma a minimí zar uma aproximação quadrática de $S\left(B_{i+1}\right)$, agora função de $\underline{p}$. Podemos, por exemplo, adotar p como o ponto de mínimo da parábola $a+b p+c p^{2}$ que passa pelos pontos de $S$ onde $p=0$ e $p=1$, e cujo declive se iguala ao de $\mathrm{S}$ em $\mathrm{p}=0$. Dal, teremos

$$
p=-b / 2 c
$$

com $a=S\left(B_{i}\right), \quad b=-G_{i}^{r} \tilde{H}_{i}^{-1} G_{i}$ e $c=S\left(B_{i+1}\right)-a-b . \quad$ Um refinamento desta idéia está elaborado a seguir.

BARD (1974) considera a fórmula iterativa

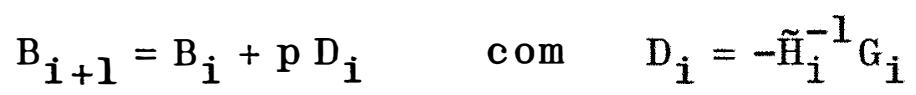

e apresenta o seguinte algoritmo para a escolha de $\underline{p}$ :

1. Coloque $\mathrm{p}_{0}=2^{-j} \quad(j=0$ na primeira iteração $)$

Calcule $S_{0}=S\left(B_{i}+p_{0} D_{i}\right)$

Se $S_{0}<S\left(B_{i}\right)$ aceite $p=p_{0}$. Caso contrário continue.

2. Calcule $d=-G_{i}^{\prime} \tilde{H}_{i}^{-1} G_{i}$

$$
\mathrm{p}^{*}=\mathrm{d} \mathrm{p}_{0}^{2} /\left[2\left(\mathrm{~S}\left(\mathrm{~B}_{\mathbf{i}}\right)-\mathrm{S}_{\mathrm{O}}-\mathrm{d} \mathrm{p}_{\mathrm{O}}\right]\right.
$$


Defina $\mathrm{p}_{1}=\max \left[0,25 \mathrm{p}_{0} ; \min \left(0,75 \mathrm{p}_{0}, \mathrm{p}^{*}\right)\right]$

$$
\mathbf{j}=\mathbf{j}+\mathbf{1}
$$

Se $\mathrm{p}_{1} \leqslant \mathrm{p}_{\min }$ aceite $\hat{\mathrm{B}}=\mathrm{B}_{\mathrm{i}}$. Caso contrário continue.

3. Retorne ao passo 1 com $p_{0}=p_{1}$.

Se, na primeira execução do passo 1 , tivermos $\mathrm{S}_{0}<\mathrm{S}\left(\mathrm{B}_{\mathbf{i}}\right)$, em vez de aceitar imediatamente $\mathrm{p}=\mathrm{p}_{0}$, podemos seguir para o passo 4 com o objetivo de tentar diminuir, ainda mais, $\mathrm{S}_{0}$.

4. Coloque $\mathbf{j}=$ maior inteiro que não supere $j / 2$.

Determine $\mathrm{p}^{*}$ como no passo 2 .

Defina $\mathrm{p}^{*}=2 \mathrm{p}_{0}$ quando $\mathrm{p}^{*}<0$

$$
\mathrm{p}_{2}=\min \left(\mathrm{p}^{*}, \mathrm{p}_{\max }\right) \text {. }
$$

5. Se $\left|p_{2}-p_{0}\right|<0,1 p_{0}$ aceite $p=p_{0}$. Caso contrário continue.

6. Calcule $\mathrm{S}_{2}=\mathrm{S}\left(\mathrm{B}_{\mathrm{i}}+\mathrm{p}_{2} \mathrm{D}_{\mathbf{i}}\right)$.

Aceite $\mathrm{p}=\mathrm{p}_{2}$ ou $\mathrm{p}=\mathrm{p}_{0}$ dependendo se $\mathrm{S}_{2}$ ou $\mathrm{S}_{0}$ é o menor.

Para a Segunda Lei de Mitscherlich, foi satis fatório adotar $p_{\min }=10^{-7}$ e $p_{\max }=10^{3}$, porém, outra alternati va é estabelecer esses valores, a cada iteração, por

$$
p_{i(\min )}=\min \left(e_{k} / D_{k i}\right) \quad \text { e } \quad p_{i(\max )}=\max \left(e_{k} / D_{k i}\right)
$$

acompanhando o critério de finalização da seção 3.3.2. 


\subsubsection{Método de Marquardt}

0 método de Marquardt se apóia na observação de que se $\mathrm{N}$ é qualquer matriz definida positiva, então $\mathrm{M}+\mathrm{qN}$ é definida positiva para o escalar q suficientemente grande, qualquer que seja a matriz M. Marquardt sugere a escolha de $\mathrm{M}=\tilde{\mathrm{H}}$, a aproximação da Hessiana do método de Gauss e $\mathrm{N}$ uma ma triz diagonal com elementos

$$
\mathrm{n}_{\mathrm{kk}}= \begin{cases}\tilde{h}_{\mathrm{kk}} & \left(\tilde{h}_{\mathrm{kk}} \neq 0\right) \\ 1 & \left(\tilde{h}_{\mathrm{kk}}=0\right)\end{cases}
$$

Portanto, a fórmula iterativa do método será:

$$
B_{i+1}=B_{i}-\left(\tilde{H}_{i}+q_{i} N_{i}\right)^{-1} G_{i}
$$

sendo $q_{i}$ determinado de modo a garantir uma matriz definida positiva.

Quando $\mathrm{q}_{\mathrm{i}} \rightarrow \infty$ a matriz $\mathrm{q}_{\mathrm{i}} \mathrm{N}_{\mathrm{i}}$ predomina sobre $\tilde{H}_{i}$ e $\left(B_{i+1}-B_{i}\right) \rightarrow\left(1 / q_{i}\right) N_{i}^{-1} G_{i}$, representando um vetor correção extremamente pequeno numa direção em declive, pois $\mathrm{N}_{i}$ é defí nida positiva. $U m q_{i}$ suficientemente grande sempre ocasiona $S\left(B_{i+1}\right)<S\left(B_{i}\right)$. Por outro lado, quando $q_{i}$ é pequeno $B_{i+1}-B_{i}$ se aproxima da correção de Gauss, $D_{i}$. Concluimos que o método de Marquardt consiste numa interpolação entre o método "da descida mais rápida" (steepest Descent) e o método de Gauss.

0 seguinte algoritmo, proposto por BARD (1974) a partir de um sugerido por Marquardt, pode ser adotado para 
a escolha de $q_{i}$ :

1. No inf́cio da primeira iteração adote $q=0,01$.

2. No início da i-ésima iteração calcule

$$
\mathrm{D}_{\mathbf{i}}=-\left(\tilde{\mathrm{H}}_{\mathbf{i}}+\mathrm{qN}_{\mathbf{i}}\right)^{-1} \mathrm{G}_{\mathbf{i}}, \mathrm{B}^{*}=\mathrm{B}_{\mathbf{i}}+\mathrm{D}_{\mathbf{i}} \text { e } \mathrm{S}^{*}=\mathrm{S}\left(\mathrm{B}^{*}\right) \text {. }
$$

3. Se $S^{*}<S\left(B_{i}\right)$, aceite $B_{i+1}=B^{*}$ e adote $q=\max \left(0,1 q ; 10^{-7}\right)$. Caso contrário continue.

4. Encontre um valor $\underline{p}$ suficientemente pequeno tal que $S\left(B_{i}+p D_{i}\right)<S\left(B_{i}\right) \quad($ ver seção 3.3.3.). Mude q por $10 q$.

3.3.5. Programação dos Métodos do Gradiente

Em primeiro lugar, escrevemos um programa, em linguagem BASIC, para o método de Marquardte, depois, sugerí mos modificações para obter os programas dos outros métodos.

\section{REM: AJUSTAMENTO DA SEGUNDA LEI DE MITSCHERLICH}

6 REM: METODO DE MARQUARDT

9 REM: M=NO.DE PARAMETROS

$1 \varnothing \mathrm{M}=4$

$2 \varnothing$ PRINT "QUAL O NO. DE OBSERVACOES"

$3 \varnothing$ INPUT $\mathrm{N}$

$35 \mathrm{DIM} \mathrm{B}(\mathrm{M}), \mathrm{B} \varnothing(\mathrm{M}), \mathrm{Bl}(\mathrm{M}), \mathrm{D}(\mathrm{M}), \mathrm{Dl}(\mathrm{M}), \mathrm{F}(\mathrm{M}), \mathrm{G}(\mathrm{M}), \mathrm{X}(\mathrm{N}), \mathrm{Y}(\mathrm{N})$

$36 \mathrm{DIM} \mathrm{H}(\mathrm{M}, \mathrm{M}), \mathrm{HI}(\mathrm{M}, \mathrm{M})$

$4 \varnothing$ PRINT "ENTRE OS PARES X,Y"

$5 \varnothing$ FOR J=1 TO N

$.6 \varnothing$ PRINT J, 
$7 \varnothing$ INPUT $X(J), Y(J)$

$8 \varnothing$ NEXT $J$

$9 \emptyset$ PRINT "ESTIMATIVAS INICIAIS DOS PARAMETROS"

$1 \varnothing \emptyset$ FOR $\mathrm{I}=1$ TO M

$11 \varnothing$ PRINT $" B(" ; \mathrm{I} ; ")="$,

$12 \varnothing$ INPUT $B(I)$

$13 \emptyset$ NEXT I

139 REM: IT=NO. DA ITERACAO

$14 \varnothing \mathrm{IT}=\varnothing$

$145 \mathrm{~J} \emptyset=\varnothing$

$147 Q=\varnothing . \varnothing 1$

149 REM: INIĊIA A ITERACAO

$15 \varnothing \mathrm{SQ}=\varnothing$

$16 \emptyset$ FOR I=1 TO M

$17 \varnothing$ FOR $\mathrm{J}=1$ TO $M$

$18 \emptyset \mathrm{H}(\mathrm{I}, \mathrm{J})=\varnothing$

$19 \emptyset$ NEXT $J$

$2 \varnothing \emptyset \mathrm{G}(\mathrm{I})=\varnothing$

$21 \varnothing$ NEXT I

219 REM: OBTEM H, G, SQ $=\mathrm{S}(\mathrm{B})$

$22 \varnothing \mathrm{Bl}=\mathrm{B}(1) * \mathrm{LOG}(1 \varnothing)$

$23 \emptyset$ FOR J=1 TO N

$24 \emptyset \mathrm{Z}=\mathrm{X}(\mathrm{J})+\mathrm{B}(2)$

$25 \varnothing \mathrm{U}=1 \varnothing[(-\mathrm{B}(3) * \mathrm{Z})$

$26 \varnothing \quad \mathrm{V}=1-\mathrm{U}$

$27 \varnothing \mathrm{W}=1 \varnothing[(-\mathrm{B}(4) * \mathrm{Z} * \mathrm{Z})$

$28 \emptyset \mathrm{F}(1)=\mathrm{V} * \mathrm{~W}$

$29 \emptyset \mathrm{F}(2)=\mathrm{Bl} * \mathrm{~W} *(\mathrm{~B}(3) * \mathrm{U}-2 * \mathrm{~B}(4) * \mathrm{Z} * \mathrm{~V})$

$3 \varnothing \emptyset \mathrm{F}(3)=\mathrm{Bl} * \mathrm{Z} * \mathrm{U} * \mathrm{~W}$

$31 \varnothing \mathrm{F}(4)=-\mathrm{B} I * \mathrm{Z} * \mathrm{Z} * \mathrm{~V} * \mathrm{~W}$

$32 \varnothing \mathrm{E}=\mathrm{Y}(\mathrm{J})-\mathrm{B}(\mathrm{I}) * \mathrm{~F}(\mathrm{I})$

$33 \emptyset \mathrm{SQ}=\mathrm{SQ}+\mathrm{E} * \mathrm{E}$

$34 \varnothing$ FOR I=1 TO M

$35 \varnothing$ FOR K=I TO M 


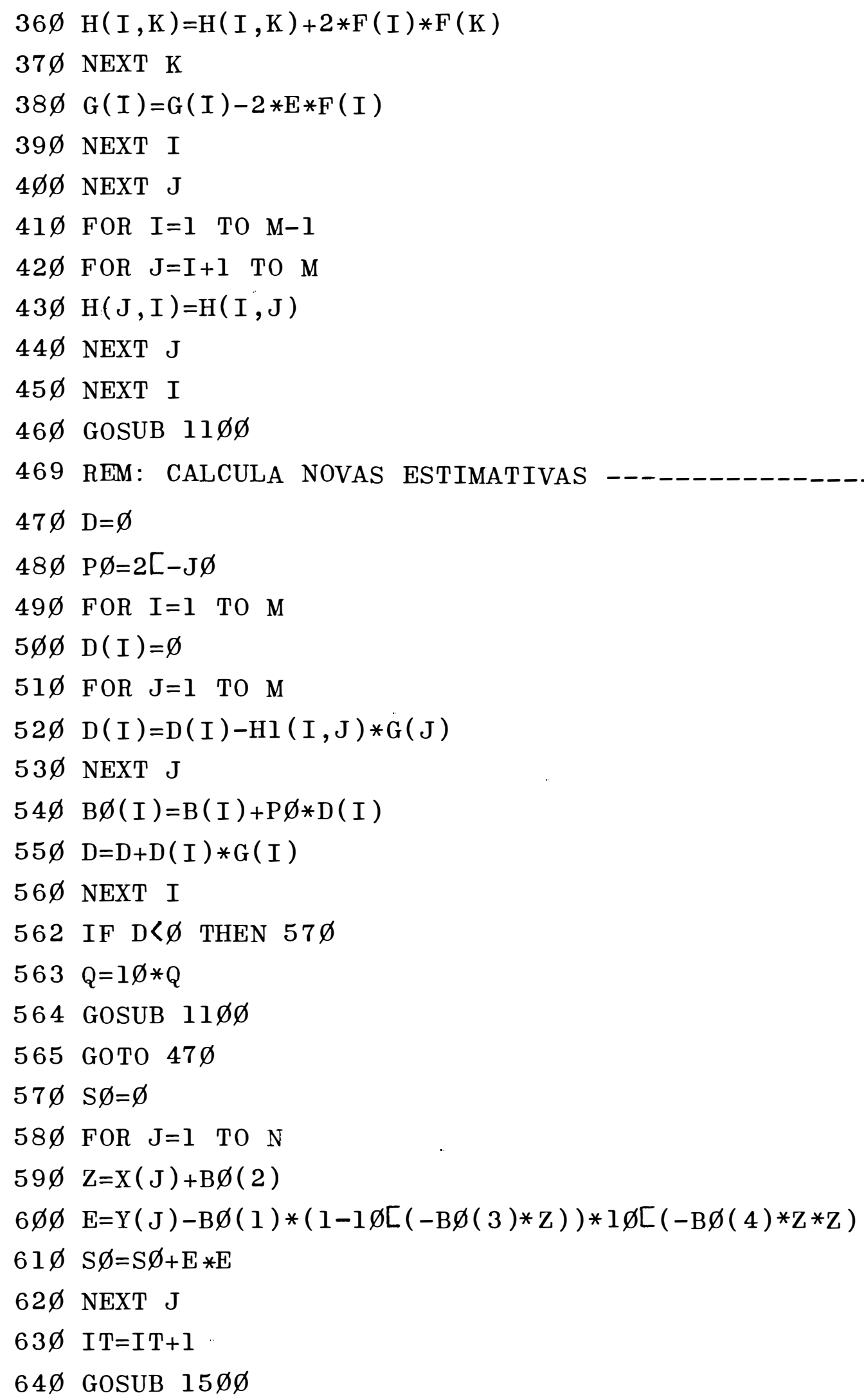


36 .

649 REM : TESTA FINALIZACAO

$65 \varnothing$ FOR $\mathrm{I}=1$ TO M

$66 \varnothing \mathrm{Dl}(\mathrm{I})=\operatorname{ABS}(\mathrm{B} \varnothing(\mathrm{I})-\mathrm{B}(\mathrm{I}))$

$67 \varnothing$ IF DI ( I ) >1 $\varnothing[-4 *(\mathrm{~B}(\mathrm{I})+1 \varnothing[-3)$ THEN $7 \varnothing \varnothing$

$68 \varnothing$ NEXT I

$69 \varnothing$ GOTO $77 \varnothing$

699 REM: PREPARA NOVA ITERACAO

$7 \varnothing \varnothing$ PRINT "ITERACAO:" ; IT

$71 \emptyset$ FOR $\mathrm{I}=1$ TO M

$72 \varnothing \mathrm{B}(\mathrm{I})=\mathrm{B} \emptyset(\mathrm{I})$

$73 \varnothing$ PRINT "B(" ; I ; ) $="$; B ( I )

$74 \varnothing$ NEXT I

$75 \varnothing$ PRINT "SQ=";S

$76 \varnothing$ GOTO $15 \varnothing$

769 REM: CALCULA ELEMENTOS PARA ANALISE

$77 \varnothing \mathrm{Ml}=\varnothing$

$78 \varnothing \mathrm{M} 2=\varnothing$

$79 \emptyset$ FOR $\mathrm{J}=1$ TO N

$8 \varnothing \varnothing \mathrm{Ml}=\mathrm{Ml}+\mathrm{Y}(\mathrm{J})$

$81 \varnothing \mathrm{M} 2=\mathrm{M} 2+\mathrm{Y}(\mathrm{J}) * \mathrm{Y}(\mathrm{J})$

$82 \varnothing \mathrm{NEXT} J$

$83 \varnothing \mathrm{C}=\mathrm{M} 1 * \mathrm{M} 1 / \mathrm{N}$

$84 \varnothing \mathrm{ST}=\mathrm{M} 2-\mathrm{C}$

$85 \varnothing \mathrm{SR}=\mathrm{ST}-\mathrm{S} \varnothing$

$86 \varnothing \mathrm{MR}=\mathrm{SR} /(\mathrm{M}-1)$

$87 \varnothing \mathrm{M} \emptyset=\mathrm{S} \emptyset /(\mathrm{N}-\mathrm{M})$

$88 \varnothing \mathrm{F}=\mathrm{MR} / \mathrm{M} \varnothing$

$89 \emptyset \mathrm{ME}=\mathrm{M} 1 / \mathrm{N}$

$9 \varnothing \varnothing \quad \mathrm{CV}=\mathrm{SQR}(\mathrm{M} \varnothing) / \mathrm{ME} * 1 \varnothing \varnothing$

$91 \varnothing \mathrm{Cl}=\mathrm{SR} / \mathrm{ST}$

$92 \emptyset \mathrm{CD}=\mathrm{Cl}-(\mathrm{M}-1) *(1-\mathrm{Cl}) /(\mathrm{N}-\mathrm{M})$ 


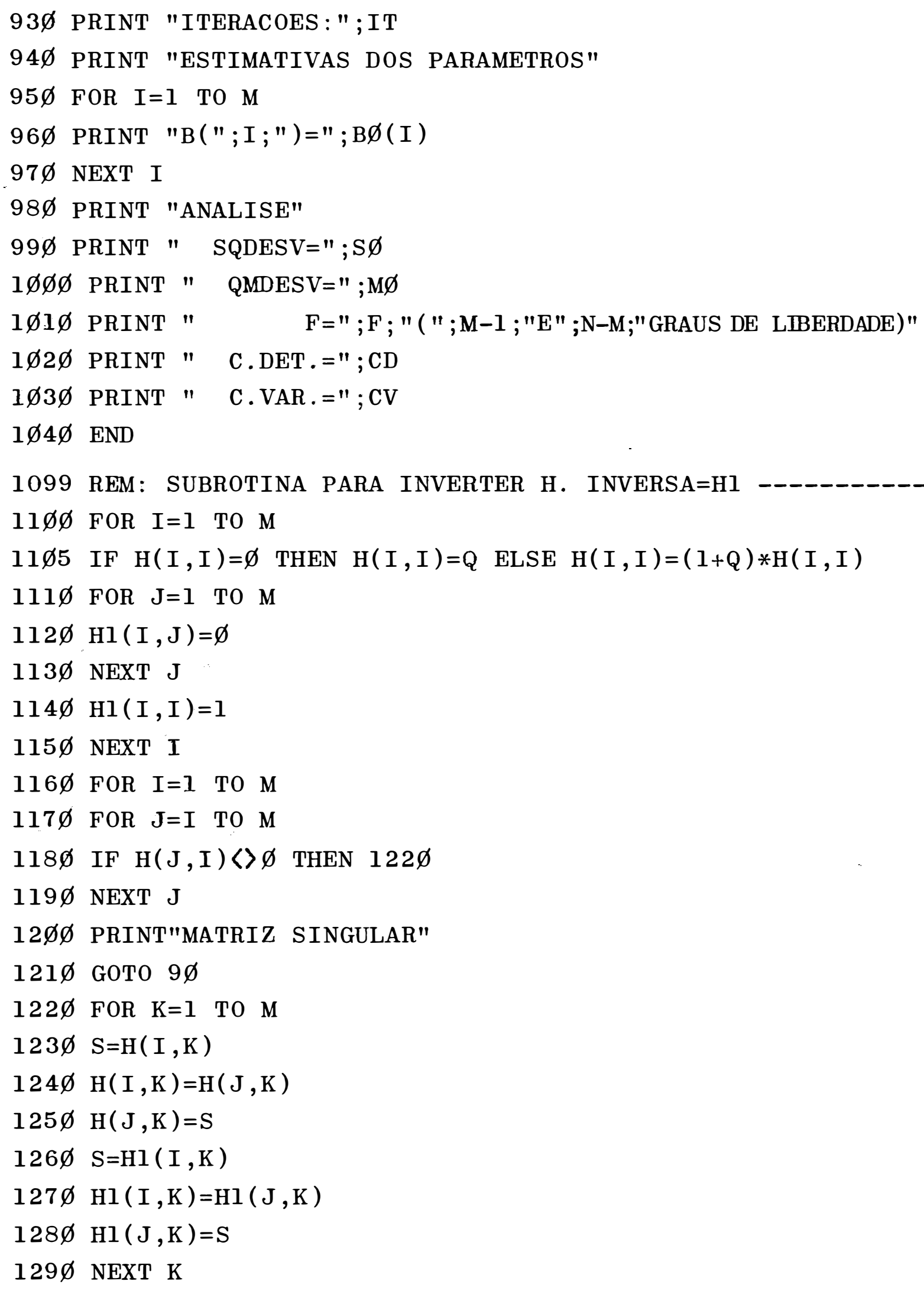


$13 \emptyset \emptyset \mathrm{T}=1 / \mathrm{H}(\mathrm{I}, \mathrm{I})$

$131 \varnothing$ FOR $\mathrm{K}=1$ TO $\mathrm{M}$

$132 \varnothing \mathrm{H}(\mathrm{I}, \mathrm{K})=\mathrm{T} * \mathrm{H}(\mathrm{I}, \mathrm{K})$

$133 \varnothing \mathrm{Hl}(\mathrm{I}, \mathrm{K})=\mathrm{T} * \mathrm{Hl}(\mathrm{I}, \mathrm{K})$

$134 \varnothing$ NEXT K

$135 \varnothing$ FOR L=1 TO M

$136 \varnothing$ IF L=I THEN $142 \varnothing$

$137 \varnothing \mathrm{T}=-\mathrm{H}(\mathrm{L}, \mathrm{I})$

$138 \varnothing$ FOR $\mathrm{K}=1$ TO $\mathrm{M}$

$139 \varnothing \mathrm{H}(\mathrm{L}, \mathrm{K})=\mathrm{H}(\mathrm{L}, \mathrm{K})+\mathrm{T} * \mathrm{H}(\mathrm{I}, \mathrm{K})$

$14 \varnothing \emptyset \mathrm{Hl}(\mathrm{L}, \mathrm{K})=\mathrm{Hl}(\mathrm{L}, \mathrm{K})+\mathrm{T} * \mathrm{Hl}(\mathrm{I}, \mathrm{K})$

$141 \varnothing$ NEXT $K$

$142 \varnothing$ NEXT L

$143 \varnothing$ NEXT I

$144 \varnothing$ RETURN

1499 REM: OBTEM SQ ACEITAVEL

$15 \varnothing \emptyset \mathrm{P}=\mathrm{D} * \mathrm{P} \emptyset * \mathrm{P} \emptyset /(2 *(\mathrm{SQ}-\mathrm{S} \emptyset+\mathrm{P} \emptyset * \mathrm{D}))$

$151 \varnothing \mathrm{J} 1=\varnothing$

$152 \varnothing$ IF $S \varnothing<S Q$ THEN $181 \varnothing$

$1525 Q=1 \varnothing * Q$

$153 \varnothing$ IF $\varnothing .75 * \mathrm{P} \varnothing<\mathrm{P}$ THEN P2 $=\varnothing .75 * \mathrm{P} \varnothing$ ELSE P2=P

$154 \varnothing$ IF $\varnothing .25 * \mathrm{P} \varnothing>\mathrm{P} 2$ THEN $\mathrm{P} 1=\varnothing .25 * \mathrm{P} \varnothing \quad$ ELSE P1=P2

$155 \varnothing$ IF $\mathrm{P} 1<=1 \varnothing[-7$ THEN $176 \varnothing$

$156 \varnothing \mathrm{J} \emptyset=\mathrm{J} \emptyset+1$

$157 \varnothing$ FOR $\mathrm{I}=1$ TO $\mathrm{M}$

$158 \emptyset \mathrm{B} 1(\mathrm{I})=\mathrm{B}(\mathrm{I})+\mathrm{P} 1 * \mathrm{D}(\mathrm{I})$

$159 \varnothing$ NEXT I

$16 \varnothing \varnothing \mathrm{Sl}=\varnothing$

$161 \varnothing$ FOR J=1 TO N

$162 \varnothing \mathrm{Z}=\mathrm{X}(\mathrm{J})+\mathrm{Bl}(2)$

$163 \varnothing \mathrm{E}=\mathrm{Y}(\mathrm{J})-\mathrm{B} 1(1) *(1-1 \varnothing[(-\mathrm{B} 1(3) * \mathrm{Z})) * 1 \varnothing[(-\mathrm{B} 1(4) * \mathrm{Z} * \mathrm{Z})$

$164 \varnothing \mathrm{Sl}=\mathrm{S} 1+\mathrm{E} * \mathrm{E}$

$165 \varnothing$ NEXT J

$166 \varnothing$ IF $S 1<S Q$ THEN $171 \varnothing$ 
39.

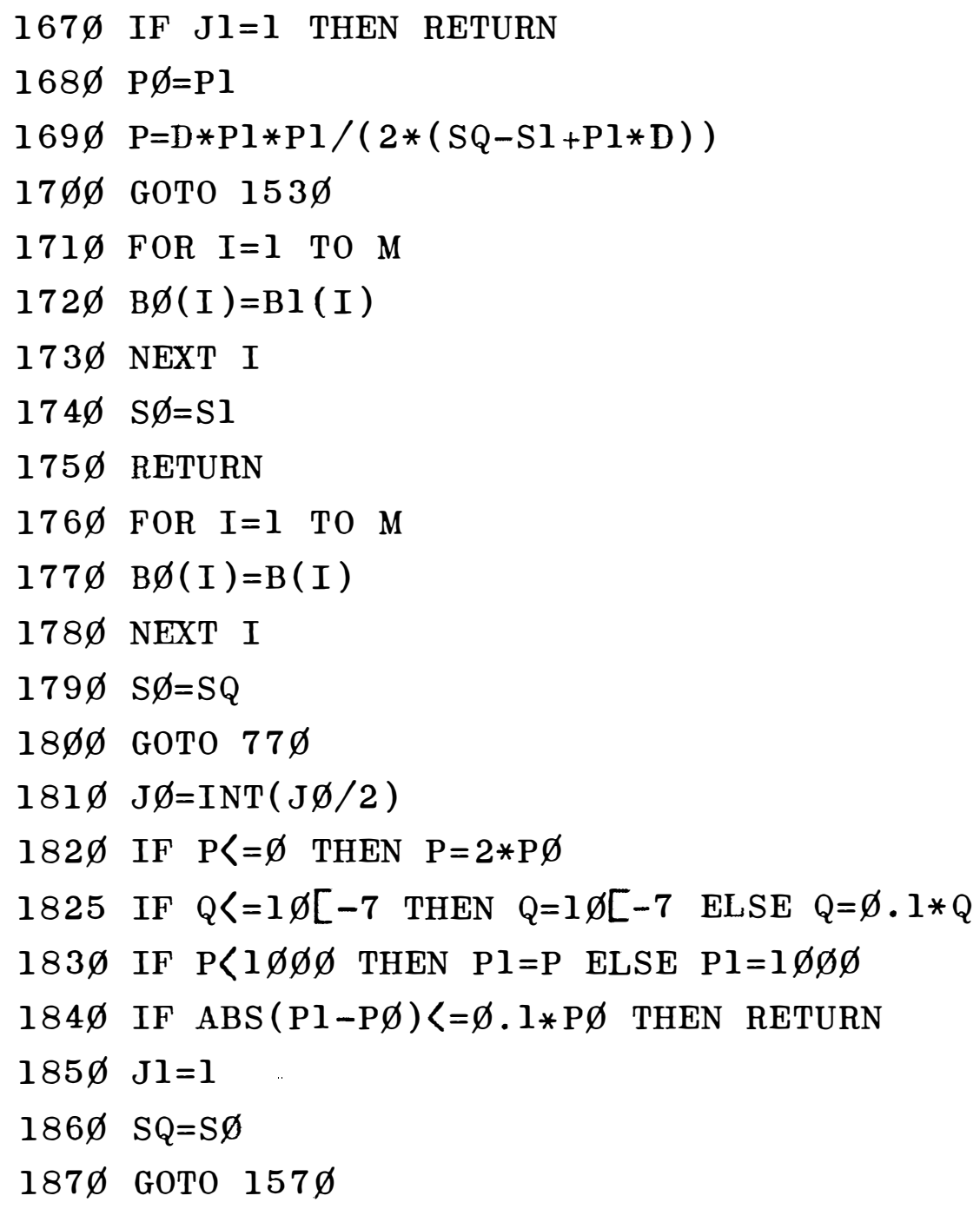

Estimados os parâmetros, se tivermos $\hat{b}_{4}>0$ haverá um ponto de máximo que pode ser determinado pelo método de Newton-Raphson. Assim, a partir de uma solução inicial $r_{0}$, pesquisamos o máximo através da fórmula iterativa

$$
r_{i+1}=r_{i}-F\left(r_{i}\right) / F^{\prime}\left(r_{i}\right)
$$

onde $F(r)=d f(r ; \hat{B}) / d r$ e $F^{\prime}(r)=d^{2} f(r ; \hat{B}) / d r^{2}$.

$\mathrm{Na}$ programação anterior devem ser anexadas as 
40 .

seguintes linhas:

$1 \varnothing 32$ IF $\mathrm{B} \emptyset(4)<=\varnothing$ THEN $1 \varnothing 38$

$1 \varnothing 33$ GOSUB $2 \varnothing \varnothing \varnothing$

$1 \emptyset 34$ PRINT "XMAX=";XM;

$1 \emptyset 35$ PRINT " YMAX $="$ YM

$1 \varnothing 36$ GOTO $1 \varnothing 4 \varnothing$

$1 \varnothing 38$ PRINT "NAO TEM MAXIMO"

1999 REM: DETERMINA O MAXIMO

$2 \varnothing \emptyset \emptyset \mathrm{R}=(\mathrm{X}(1)+\mathrm{X}(\mathrm{N})) / 2$

$2 \emptyset 1 \emptyset \mathrm{Al}=(\mathrm{B} \emptyset(3)+2 * \mathrm{~B} \emptyset(4) *(\mathrm{R}+\mathrm{B} \emptyset(2))) * 1 \varnothing[(-\mathrm{B} \emptyset(3) *(\mathrm{R}+\mathrm{B} \emptyset(2)))$

$2 \varnothing 2 \varnothing \mathrm{A} 2=2 * \mathrm{~B} \emptyset(4) *(\mathrm{R}+\mathrm{B} \emptyset(2))$

$2 \varnothing 3 \emptyset \mathrm{A} 3=1-1 \varnothing[(-\mathrm{B} \varnothing(3) *(\mathrm{R}+\mathrm{B} \emptyset(2)))$

$2 \emptyset 4 \emptyset \mathrm{NU}=\mathrm{A} 1-\mathrm{A} 2$

$2 \not 5 \varnothing \mathrm{DE}=-2 * \mathrm{~B} \emptyset(4) * \mathrm{~A} 3-\mathrm{B} \emptyset(3) * \mathrm{LOG}(1 \varnothing) * \mathrm{Al}$

$2 \varnothing 6 \varnothing \mathrm{R} \emptyset=\mathrm{R}-\mathrm{NU} / \mathrm{DE}$

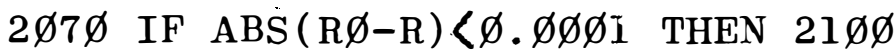

$2 \varnothing 8 \varnothing \mathrm{R}=\mathrm{R} \varnothing$

$2 \varnothing 9 \varnothing$ GOTO $2 \varnothing 1 \varnothing$

$21 \varnothing \emptyset \mathrm{XM}=\mathrm{R} \varnothing$

$211 \varnothing \mathrm{Z}=\mathrm{XM}+\mathrm{B} \emptyset(2)$

$212 \varnothing \mathrm{YM}=\mathrm{B} \varnothing(1) *(1-1 \varnothing[(-\mathrm{B} \varnothing(3) * \mathrm{Z})) * 1 \varnothing[(-\mathrm{B} \emptyset(4) * \mathrm{Z} * \mathrm{Z})$

$213 \varnothing$ RETURN

( $[$ : símbolo para a exponenciação)

Eliminando na programação anterior as linhas 147,562 a $565,11 \varnothing 5$ e 1825 obtemos a do método de Gauss. Quanto à programação do método de Newton, além destas supres sões, devemos calcular entre as linhas de 280 a 320 as deriva 
das parciais de segunda ordem, dadas na seção 3.3.1., através de um vetor $F l(I, K)$ e alterar a linha $36 \emptyset$ para:

$$
360 \mathrm{H}(\mathrm{I}, \mathrm{K})=\mathrm{H}(\mathrm{I}, \mathrm{K})+2 * \mathrm{~F}(\mathrm{I}) * \mathrm{~F}(\mathrm{~K})-2 * \mathrm{E} * \mathrm{Fl}(\mathrm{I}, \mathrm{K})
$$

Como neste método $\mathrm{p}=1$, devemos modificar a linha $48 \emptyset$ para:

$$
48 \varnothing P \varnothing=1
$$

e suprimir a linha $64 \varnothing$, portanto, também a sub-rotina corres pondente. 
42 .

4. RESUltados E Discussões

4.1. Estimativas dos Parâmetros do Modelo Quadrático

0 ajustamento das equações quadráticas aos da dos experimentais de maturação da cana-de-açúcar da Tabela l, comentados em 3.1., não apresentou qualquer dificuldade. As estimativas dos parâmetros, expostas na Tabela 2 , foram obtí das pela programação da seção 3.2.4. A Tabela 4 mostra as so mas de quadrados dos desvios(SQDesvios)e os valores máximos de Pol\% da cana $\left(\mathrm{Y}_{\max }\right)$ com as correspondentes épocas $\left(\mathrm{X}_{\max }\right)$, que variaram entre meados de agosto e início de outubro, quando adotamos o modelo Quadrático para estudar as 27 variedades quanto ao problema de maturação. Ao mesmo tempo, esses resul tados podem ser confrontados, pela Tabela 4, com os da Segun da Lei de Mitscherlich. Uma discussão a respeito é apresenta da na seção 4.3 . 
Tabela 1. Resultados médios de Pol\% da cana, obtidos na região de Sertãozinho (S.P.), em 1970 ( 1 a 19) e na Usina São João, Araras (S.P.), em 1979 (20 a 27 ).

\begin{tabular}{lllllllll}
\hline & No & Variedade & \multicolumn{10}{c}{ Meses } \\
\cline { 2 - 8 } & 1 & 2 & 3 & 4 & 5 & 6 & 7 & 8
\end{tabular}
maio junho julho agos. set. out. nov. dez.

$1 \quad$ CB-40-13 $13,98 \quad 14,87 \quad 16,03 \quad 17,04 \quad 17,75 \quad 17,89 \quad 15,93 \quad 16,59$

2 CB-40-77 $8,57 \quad 12,62 \quad 14,83 \quad 15,66 \quad 17,72 \quad 17,76 \quad 13,72 \quad 17,69$

3 CB-41-76 9,10 $13,07 \quad 15,41 \quad 15,91 \quad 17,32 \quad 16,75 \quad 15,54 \quad 16,43$

$\begin{array}{llllllllll}4 & \mathrm{CB}-46-47 & 11,64 & 14,82 & 16,84 & 16,80 & 17,52 & 18,08 & 16,47 & 16,72\end{array}$

5 CB-49-260 $10,41 \quad 13,83 \quad 14,19 \quad 16,98 \quad 16,75 \quad 16,29 \quad 16,67 \quad 16,40$

6 CB-56-121 $12,42 \quad 13,97 \quad 13,19 \quad 16,39 \quad 15,96 \quad 17,51 \quad 14,83 \quad 15,51$

7 CB-56-126 $10,8614,38 \quad 14,86 \quad 16,04 \quad 16,9016,36 \quad 16,5016,68$

8 CB-56-164 12,80 14,47 15,12 $16,32 \quad 16,92 \quad 16,08 \quad 15,76 \quad 16,02$

$\begin{array}{llllllllllll}9 & \mathrm{C} 0-178 & 10,02 & 12,03 & 14,11 & 15,78 & 15,80 & 17,07 & 14,97 & 15,18\end{array}$

$10 \mathrm{C} 0-740 \quad 9,04 \quad 13,33 \quad 15,59 \quad 17,12 \quad 17,30 \quad 18,15 \quad 17,71 \quad 17,64$

$11 \quad \mathrm{C} 0-775 \quad 10,79 \quad 13,85 \quad 15,29 \quad 20,42 \quad 19,76 \quad 18,70 \quad 16,54 \quad 16,60$

12 IAC-48-31 $10,69 \quad 14,41 \quad 16,28 \quad 17,30 \quad 17,53 \quad 17,18 \quad 14,27 \quad 14,57$

13 IAC-51-201 8,90 $11,48 \quad 14,60 \quad 15,71 \quad 16,12 \quad 15,67 \quad 14,12 \quad 14,57$

14 IAC-51-264 10,14 12,24 $15,81 \quad 17,54 \quad 17,57 \quad 16,66 \quad 16,29 \quad 15,30$

15 IAC-51-285 9,81 13,12 $15,14 \quad 17,52 \quad 17,34 \quad 16,8014,81 \quad 15,14$

16 IAC-52-130 $11,18 \quad 13,88 \quad 15,51 \quad 17,36 \quad 17,57 \quad 15,94 \quad 15,84 \quad 14,51$

17 IAC-52-172 $7,87 \quad 11,28 \quad 12,71 \quad 14,81 \quad 15,83 \quad 16,28 \quad 15,70 \quad 14,93$

18 IAC-52-179 8,90 $11,16 \quad 13,59 \quad 15,96 \quad 15,61 \quad 16,55 \quad 16,16 \quad 13,97$

19 IAC-80-326 9,20 12,63 15, o7 $16,41 \quad 16,32 \quad 16,55 \quad 17,42 \quad 16,33$

20 CB-40-13 13,07 $14,90 \quad 15,92 \quad 16,45 \quad 16,92 \quad 16,29$

21 CB-41-76 $11,11 \quad 11,5913,2014,91 \quad 14,3214,21$

22 CB.53-98 $10,28 \quad 12,48 \quad 12,81 \quad 14,18 \quad 14,99 \quad 14,59$

23 CB.47-355 10,20 $12,37 \quad 13,57 \quad 15,5016,23 \quad 15,54$

24 CP.51-22 $10,84 \quad 12,16 \quad 13,87 \quad 15,5915,37 \quad 15,09$

25 IAC.48-65 $10,6512,66 \quad 13,93 \quad 15,82 \quad 15,57 \quad 14,98$

26 IAC-52-150 $10,58 \quad 12,9914,93 \quad 15,25 \quad 16,03 \quad 16,02$

27 NA-56-79 $12,61 \quad 14,58 \quad 15,42 \quad 16,12 \quad 16,8916,33$ 
Tabela 2. Estimativas dos parâmetros do modelo Quadrático.

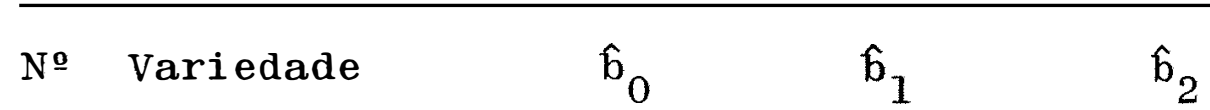

\begin{tabular}{|c|c|c|c|c|}
\hline 1 & $C B-40-13$ & 11,8996 & 2,0119 & $-0,1841$ \\
\hline 2 & CB- $40-77$ & 5,6584 & 3,8748 & $-0,3245$ \\
\hline 3 & CB-41-76 & 6,3016 & 3,7856 & $-0,3292$ \\
\hline 4 & CB $-46-47$ & 9,3702 & 3,0682 & $-0,2771$ \\
\hline 5 & $C B-49-260$ & 8,1150 & 2,9862 & $-0,2495$ \\
\hline 6 & $\mathrm{CB}-56-121$ & 10,2747 & 2,0403 & $-0,1758$ \\
\hline 7 & CB-56-126 & 9,1868 & 2,5339 & $-0,2065$ \\
\hline 8 & $\mathrm{CB}-56-164$ & 11,2119 & 1,8775 & $-0,1657$ \\
\hline 9 & C0-178 & 6,8814 & 3,2846 & $-0,2860$ \\
\hline 10 & $\mathrm{C} 0-740$ & 5,9585 & 4,0453 & $-0,3305$ \\
\hline 11 & $\mathrm{C} 0-775$ & 5,8720 & 5,0841 & $-0,4807$ \\
\hline 12 & IAC $-48-31$ & 7,5409 & 4,0479 & $-0,4109$ \\
\hline 13 & IAC $-51-201$ & 5,5055 & 3,8908 & $-0,3576$ \\
\hline 14 & IAC $-51-264$ & 6,1116 & 4,2563 & $-0,3949$ \\
\hline 15 & IAC $-51-285$ & 6,2210 & 4,2201 & $-0,4020$ \\
\hline 16 & IAC $-52-130$ & 8,0616 & 3,5968 & $-0,3539$ \\
\hline 17 & IAC $-52-172$ & 4,4316 & 3,8620 & $-0,3190$ \\
\hline 18 & IAC-52-179 & 4,8561 & 4,0820 & $-0,3623$ \\
\hline 19 & IAC- $-80-326$ & 6,3595 & 3,5962 & $-0,2961$ \\
\hline 20 & $\mathrm{CB}-40-13$ & 10,9060 & 2,4608 & $-0,2589$ \\
\hline 21 & $\mathrm{CB}-41-76$ & 8,7250 & 2,1944 & $-0,2098$ \\
\hline 22 & CB-53-98 & 8,3300 & 2,2550 & $-0,1979$ \\
\hline 23 & $\mathrm{CB}-47-355$ & 7,1840 & 3,1713 & $-0,2889$ \\
\hline 24 & CP-51-22 & 7,9400 & 2,8965 & $-0,2807$ \\
\hline 25 & IAC $-48-65$ & 7,5280 & 3,3070 & $-0,3407$ \\
\hline 26 & IAC $-52-150$ & 7,8460 & 3,1394 & $-0,2989$ \\
\hline 27 & NA-56-79 & 10,5470 & 2,3657 & $-0,2309$ \\
\hline
\end{tabular}


4.2. Estimativas dos Parâmetros do Modelo da Segunda Lei de Mitscherlich.

0 ajustamento da Segunda Lei de Mitscherlich aos dados experimentais pelo método de Marquardt, conforme a programação da seção 3.3.5., pôde ser feito, para toda varie dade estudada, a partir do valor inicial $b_{11}$ igual ao menor in teiro maior do que o $\mathrm{Y}_{j}$ máximo e de $\mathrm{b}_{21}=\mathrm{b}_{31}=\mathrm{b}_{41}=0$. Deste modo, na segunda iteração houve uma modificaçã் apenas em $b_{31}$ e só a partir da terceira iteração ocorreram modificações simulta neamente nos quatro valores. Em muitos casos, depois de poucas iterações, foi alcançada uma soma de quadrados dos desvios ra zoavelmente pequena, mas sua diminuição exigiu continuar com um número grande de iterações, aumentando considerave lmente a estimativa de $b_{1}$, diminuindo a de $b_{3}$ e alterando pouco as estimativas tanto de $b_{2}$ como de $b_{4}$. Isso permite "imaginar" uma superfície de somas de quadrados estreita e alongada, na direção do eixo $b_{1}$, que causa a convergência lenta observada. Na Tabela 6 do apêndice, onde fica indicada a forma da superfície, temos as estimativas obtidas por três critérios de finali-

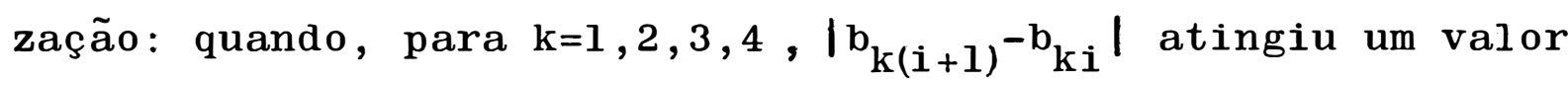
menor do que

$$
\begin{aligned}
& 1 \text { o) } 10^{-2}\left(\mathrm{~b}_{\mathrm{ki}}+10^{-3}\right) \\
& 2 \text { o }) 10^{-3}\left(\mathrm{~b}_{\mathrm{ki}}+10^{-3}\right) \\
& \text { 3 ) } 10^{-4}\left(\mathrm{~b}_{\mathrm{ki}}+10^{-3}\right)
\end{aligned}
$$


Modificamos, então, a programação do método de Marquardt, dada em 3.3.5., adotando o primeiro critério como maneira de finalização e se, em alguma iteração, $b_{1 i}$ atinge um valor igual ou maior do que o dobro do inicial a pesquisa prossegue com o dobro de $b_{1 i}$ e com $b_{3 i}$ reduzido à metade. Quan do o novo valor inicial $\left(b_{1 i}\right)$ duplica novamente, o critério é repetido. No programa devemos eliminar a linha $12 \varnothing$ e modificar ou introduzir as seguintes linhas:

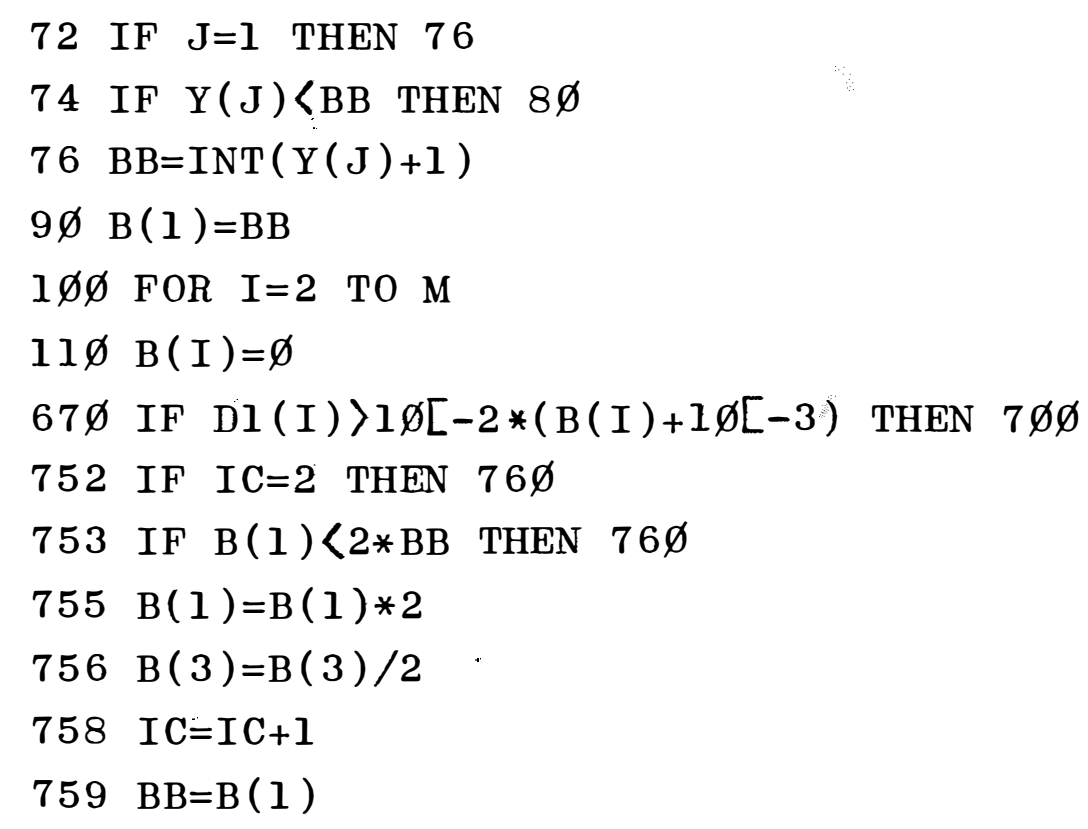

As estimativas obtidas deste modo, e aceitas neste trabalho, estão na Tabela 3 .

Para efeito de comparação, e visualização, te mos na Tabela 4 as somas de quadrados dos desvios e os valores máximos de Pol\% da cana comas respectivas épocas e, a seguir, os gráficos das duas equações ajustadas, de Mitscherlich e Quadrática, para cada variedade. 
Tabela 3. Estimativas dos parâmetros do modelo da Segunda Lei de Mitscherlich.

№ $\begin{array}{llllll}\text { Variedade } & \text { Iterações } & \hat{b}_{1} & \hat{b}_{2} & \hat{b}_{3} & \hat{b}_{4}\end{array}$

\begin{tabular}{|c|c|c|c|c|c|c|}
\hline 1 & $C B-40-13$ & 8 & 32,6154 & 3,4547 & 0,057974 & 0,001469 \\
\hline 2 & $\mathrm{CB}-40-77$ & 8 & 18,4890 & 0,1217 & 0,240052 & 0,000747 \\
\hline 3 & $C B-41-76$ & 7 & 18,5599 & 0,1573 & 0,252979 & 0,000918 \\
\hline 4 & $\mathrm{CB}-46-47$ & 6 & 19,6116 & 0,6359 & 0,241455 & 0,000933 \\
\hline 5 & $C B-49-260$ & 6 & 19,1377 & 0,8190 & 0,190927 & 0,000771 \\
\hline 6 & $C B-56-121$ & 8 & 76,3991 & 3,8597 & 0,017167 & 0,001901 \\
\hline 7 & CB-56-126 & 7 & 16,6087 & 0,3824 & 0,337209 & $-0,000023$ \\
\hline 8 & CB-56-164 & 6 & 21,3923 & 2,5092 & 0,117660 & 0,000970 \\
\hline 9 & $\mathrm{CO}-178$ & 16 & 80,3807 & 1,8352 & 0,021087 & 0,003206 \\
\hline 10 & $\mathrm{CO}-740$ & 7 & 19,4308 & 0,1763 & 0,232098 & 0,000545 \\
\hline 11 & $\mathrm{CO}-775$ & 42 & 169,6710 & 1,0559 & 0,013920 & 0,005218 \\
\hline 12 & IAC $-48-31$ & 6 & 26,1400 & 0,6593 & 0,141309 & 0,003251 \\
\hline 13 & $I A C-51-201$ & 6 & 24,7750 & 0,6158 & 0,118399 & 0,002751 \\
\hline 14 & IAC-5I-264 & 17 & 78,1376 & 1,2021 & 0,027842 & 0,004315 \\
\hline 15 & IAC $-51-285$ & 6 & 31,0080 & 0,7556 & 0,095464 & 0,003407 \\
\hline 16 & IAC-52-130 & 11 & 47,3563 & 1,5283 & 0,048852 & 0,003697 \\
\hline 17 & IAC $-52-172$ & 16 & 79,0446 & 1,1351 & 0,022241 & 0,003537 \\
\hline 18 & IAC-52-179 & 16 & 130,8260 & 1,1924 & 0,013773 & 0,004249 \\
\hline 19 & IAC-80-326 & 6 & 18,7998 & 0,3635 & 0,213554 & 0,000683 \\
\hline 20 & $C B-40-13$ & 16 & 28,7530 & 2,6474 & 0,077912 & 0,001886 \\
\hline 21 & $C B-41-76$ & 8 & 82,9975 & 3,3158 & 0,015545 & 0,002522 \\
\hline 22 & $C B-53-9.8$ & 14 & 26,0169 & 2,5694 & 0,065146 & 0,001451 \\
\hline 23 & $\mathrm{CB}-47-355$ & 20 & 78,3646 & 2,0968 & 0,020619 & 0,003025 \\
\hline 24 & $C P-51-22$ & 14 & 75,9512 & 2,4050 & 0,020515 & 0,003090 \\
\hline 25 & IAC- $48-65$ & 12 & 76,4359 & 2,0183 & 0,022752 & 0,003783 \\
\hline 26 & IAC-52-150 & 6 & 17,9106 & 0,9655 & 0,198361 & 0,000610 \\
\hline 27 & $\mathrm{NA}-56-79$ & 16 & 26,9168 & 2,7022 & 0,079488 & 0,001519 \\
\hline
\end{tabular}


48.

Tabela 4. Valores máximos de Pol\% da cana ( $\left.\mathrm{Y}_{\max }\right)$ com a respectiva época $\left(X_{\max }\right)$ e as somas de quadrados dos desvios (SQDesvios), para os dois modelos.

\begin{tabular}{|c|c|c|c|c|c|c|c|}
\hline \multirow{2}{*}{ No } & \multirow{2}{*}{ Variedade } & \multicolumn{3}{|c|}{ Mitscherlich } & \multicolumn{3}{|c|}{ Quadrático } \\
\hline & & SQDesvios & $\mathrm{X}_{\max }$ & $\mathrm{Y}_{\max }$ & SQDesvios & $\mathrm{X}_{\max }$ & $\mathrm{Y}_{\max }$ \\
\hline 1 & $C B-40-13$ & 1,9890 & 5,35 & 17,35 & 1,8882 & 5,47 & 17,40 \\
\hline 2 & $\mathrm{CB}-40-77$ & 12,6863 & 5,89 & 16,75 & 15,1453 & 5,97 & 17,23 \\
\hline 3 & $\mathrm{CB}-41-76$ & 1,5364 & 5,42 & 16,70 & 3,6619 & 5,75 & 17,18 \\
\hline 4 & $\mathrm{CB}-46-47$ & 1,1797 & 5,06 & 17,52 & 2,2688 & 5,54 & 17,86 \\
\hline 5 & $\mathrm{CB}-49-260$ & 2,0488 & 5,92 & 16,74 & 2,7112 & 5,98 & 17,05 \\
\hline 6 & CB-56-121 & 6,5791 & 5,82 & 16,12 & 6,4313 & 5,80 & 16,19 \\
\hline 7 & CB-56-126 & 0,7702 & - & - & 2,0216 & 6,13 & 16,96 \\
\hline 8 & CB-56-164 & 0,7793 & 5,44 & 16,42 & 0,9397 & 5,67 & 16,53 \\
\hline 9 & $\mathrm{C} 0-178$ & 1,8263 & 5,66 & 16,20 & 1,8383 & 5,74 & 16,31 \\
\hline 10 & $\mathrm{C} 0-740$ & 0,2347 & 6,39 & 17,86 & 1,8380 & 6,12 & 18,34 \\
\hline 11 & $\mathrm{C} 0-775$ & 8,2868 & 5,08 & 19,27 & 8,9775 & 5,29 & 19,32 \\
\hline 12 & IAC-48-31 & 2,1212 & 4,44 & 17,42 & 3,5966 & 4,93 & 17,51 \\
\hline 13 & IAC-51-201 & 1,7208 & 5,11 & 15,90 & 2,6940 & 5,44 & 16,09 \\
\hline 14 & IAC-51-264 & 1,7742 & 5,18 & 17,51 & 2,2692 & 5,39 & 17,58 \\
\hline 15 & IAC $-51-285$ & 2,1741 & 4,91 & 17,17 & 3,2299 & 5,25 & 17,30 \\
\hline 16 & IAC $-52-130$ & 1,1412 & 4,82 & 17,15 & 1,5425 & 5,08 & 17,20 \\
\hline 17 & IAC $-52-172$ & 0,4969 & 6,00 & 15,98 & 0,4111 & 6,05 & 16,12 \\
\hline 18 & IAC-52-179 & 1,8853 & 5,58 & 16,14 & 1,4423 & 5,63 & 16,36 \\
\hline 19 & IAC $-80-326$ & 0,8118 & 6,18 & 16,87 & 1,8778 & 6,07 & 17,28 \\
\hline 20 & CB-40-13 & 0,0991 & 4,77 & 16,66 & 0,0759 & 4,75 & 16,75 \\
\hline 21 & CB-41-76 & 1,3978 & 5,26 & 14,31 & 1,2953 & 5,23 & 14,46 \\
\hline 22 & CB-5 3-98 & 0,6120 & 6,00 & 14,73 & 0,5834 & 5,70 & 14,76 \\
\hline 23 & $\mathrm{CB}-47-355$ & 0,6477 & 5,61 & 15,88 & 0,6038 & 5,49 & 15,89 \\
\hline 24 & $C P-51-22$ & 0,9224 & 5,23 & 15,19 & 0,6625 & 5,16 & 15,41 \\
\hline 25 & IAC $-48-65$ & 0,7936 & 4,88 & 15,32 & 0,5247 & 4,85 & 15,55 \\
\hline 26 & IAC-52-150 & 0,1739 & 6,02 & 16,04 & 0,2905 & 5,25 & 16,09 \\
\hline 27 & NA-56-79 & 0,2244 & 5,25 & 16,54 & 0,2073 & 5,12 & 16,61 \\
\hline
\end{tabular}


Figuras de (1) a (27). Gráficos da equações ajustadas :

Mitscherlich

- - - Quadrática

com as épocas de máximo Pol\% da cana, $M$ e $Q$ respectivamente.

- ponto experimental
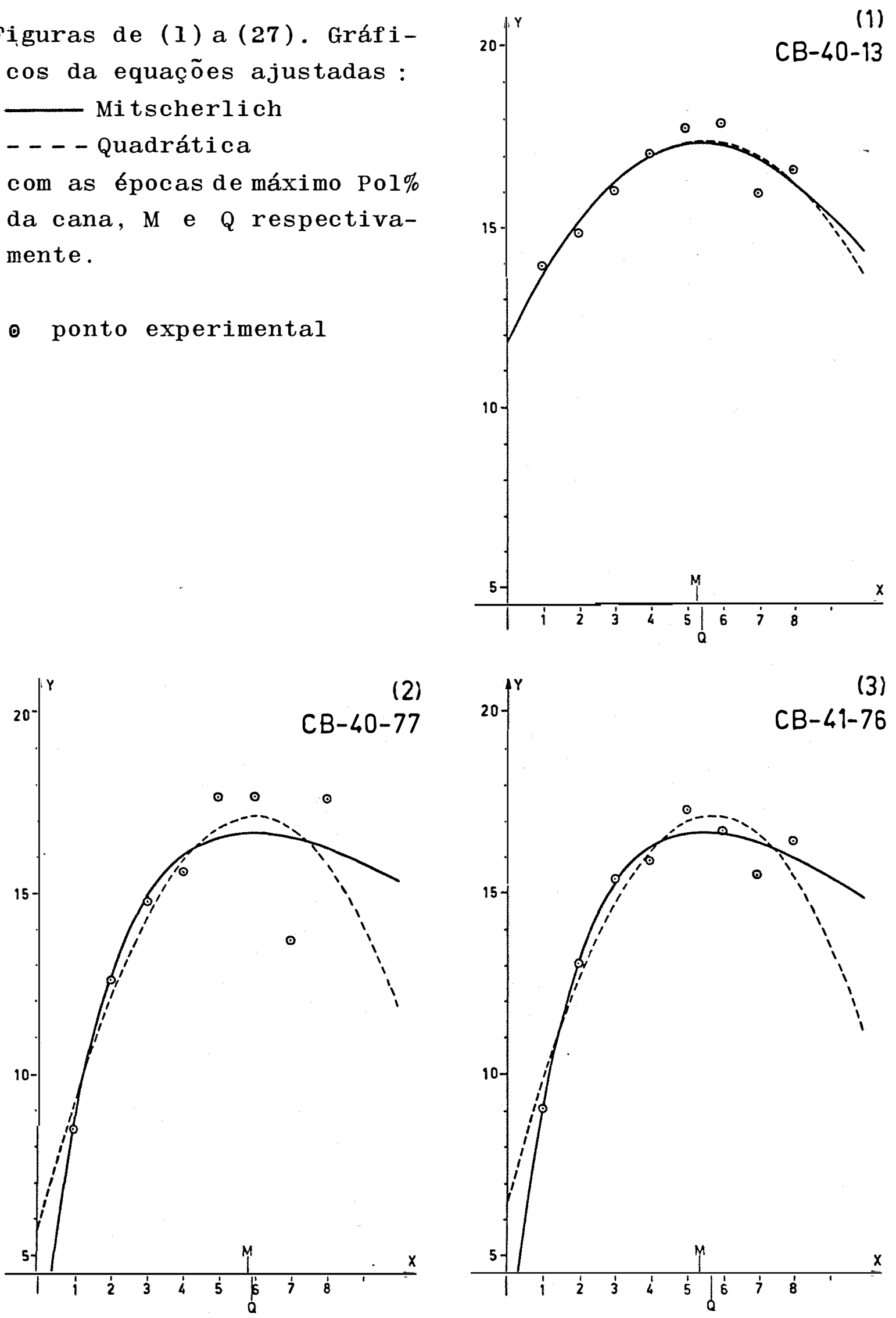
50.
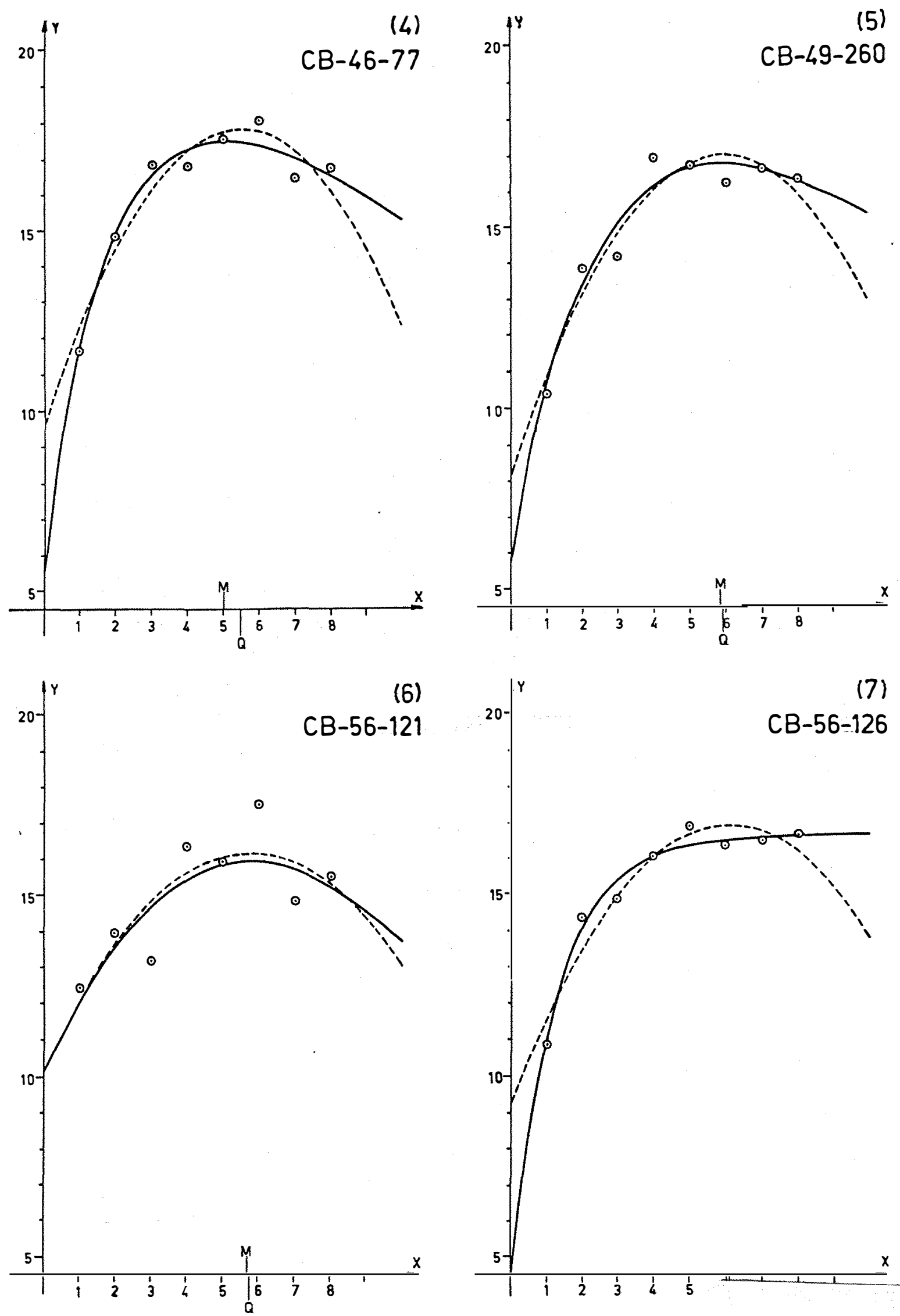
51.
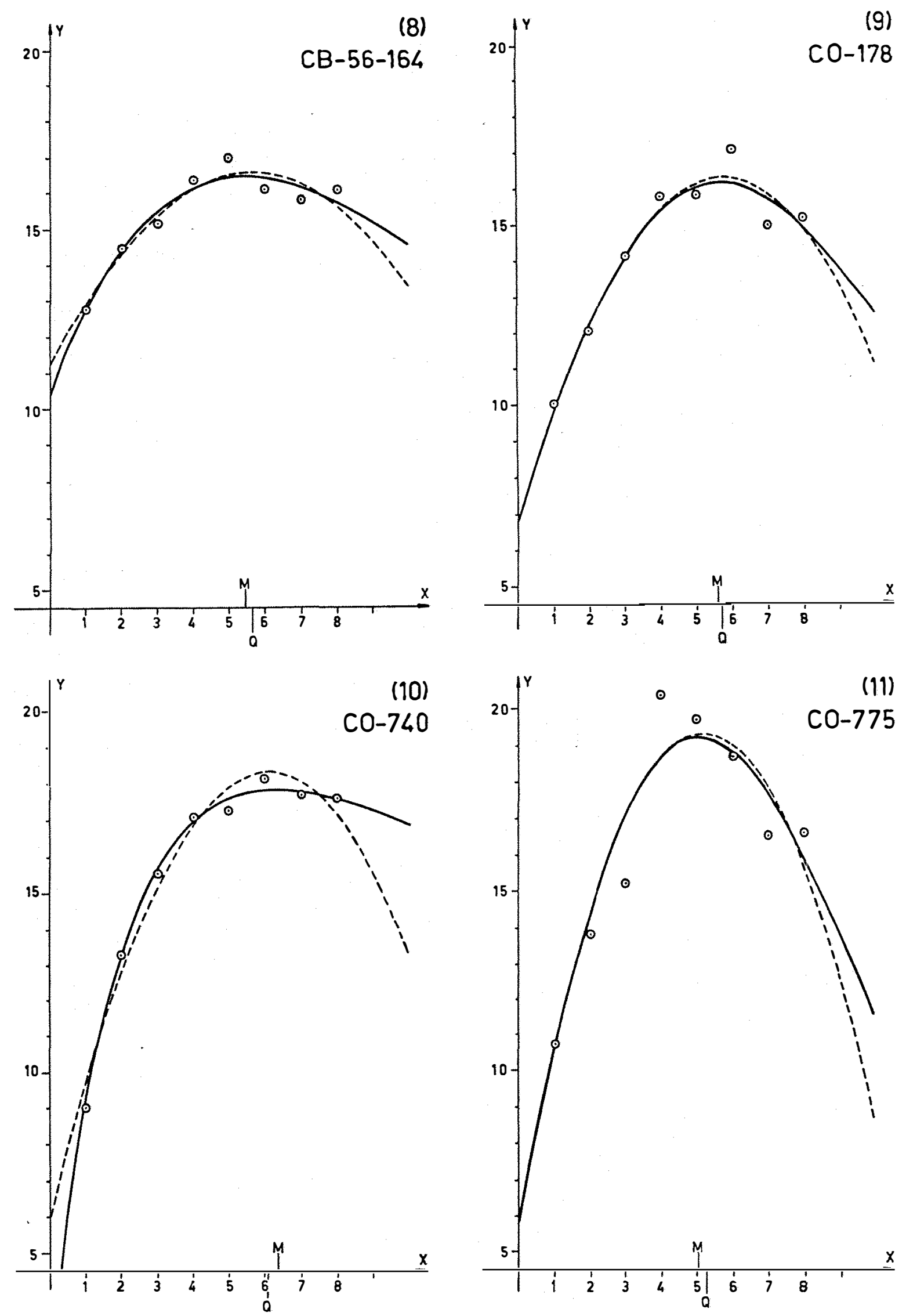
52.
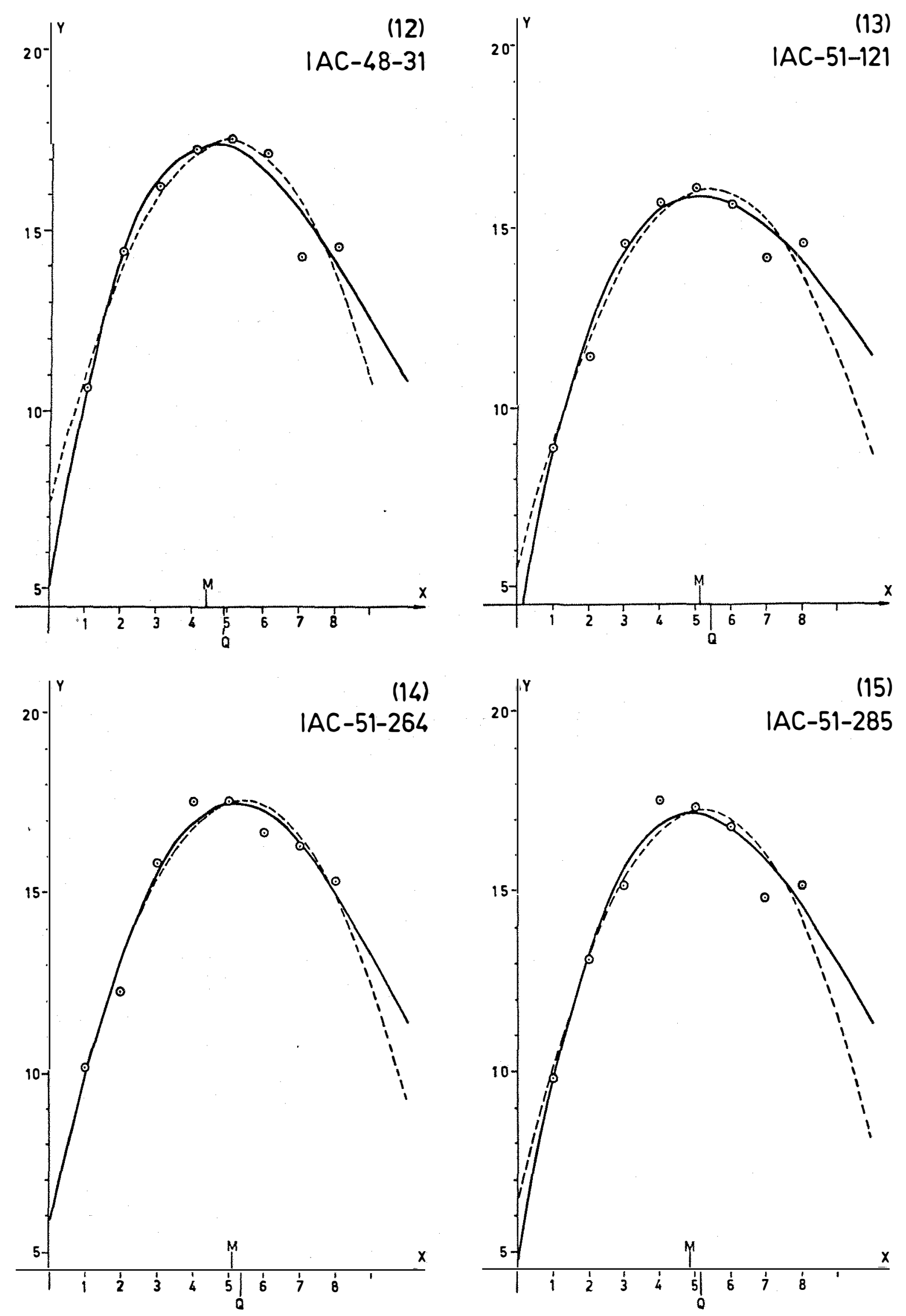
53.
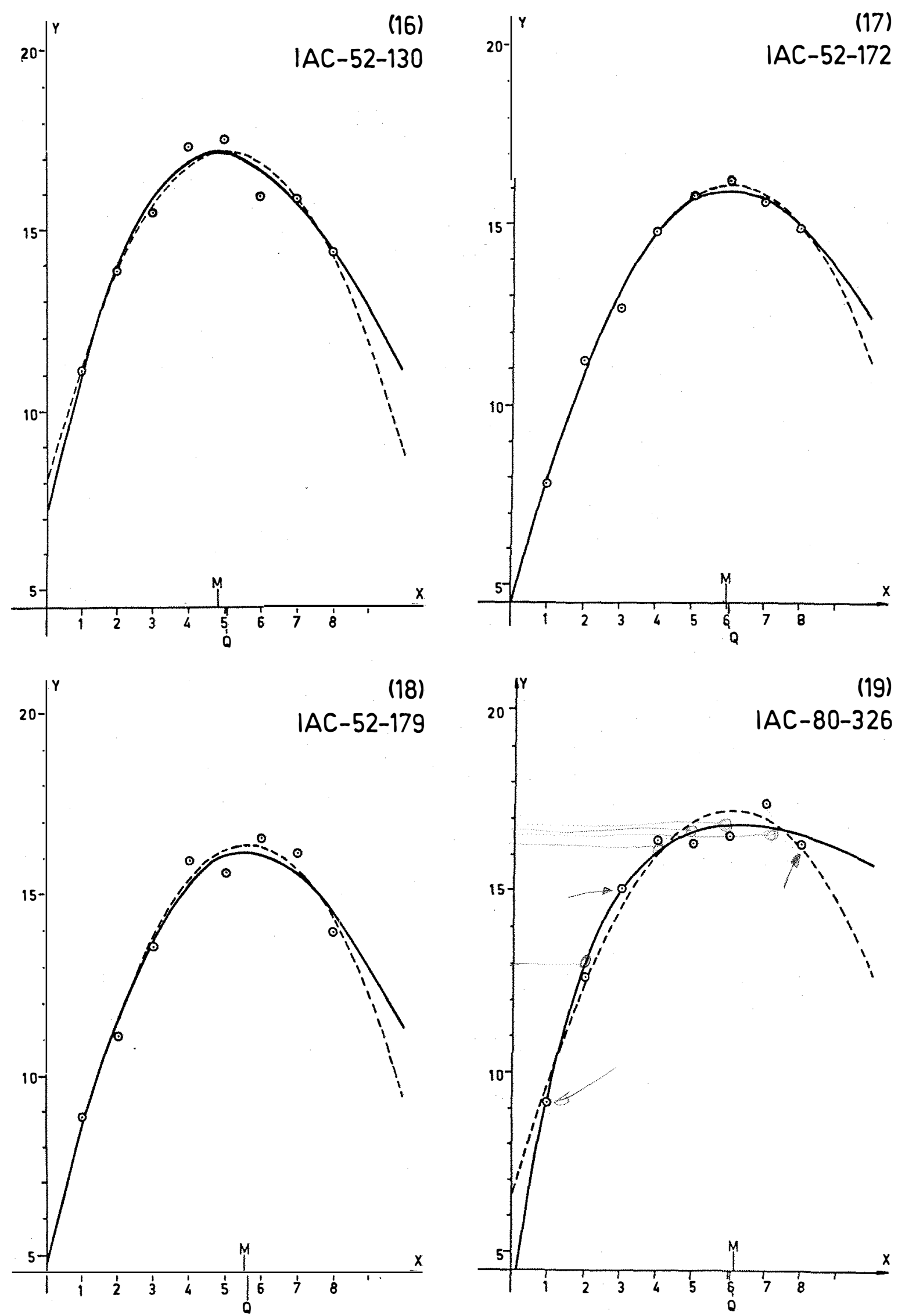
54.
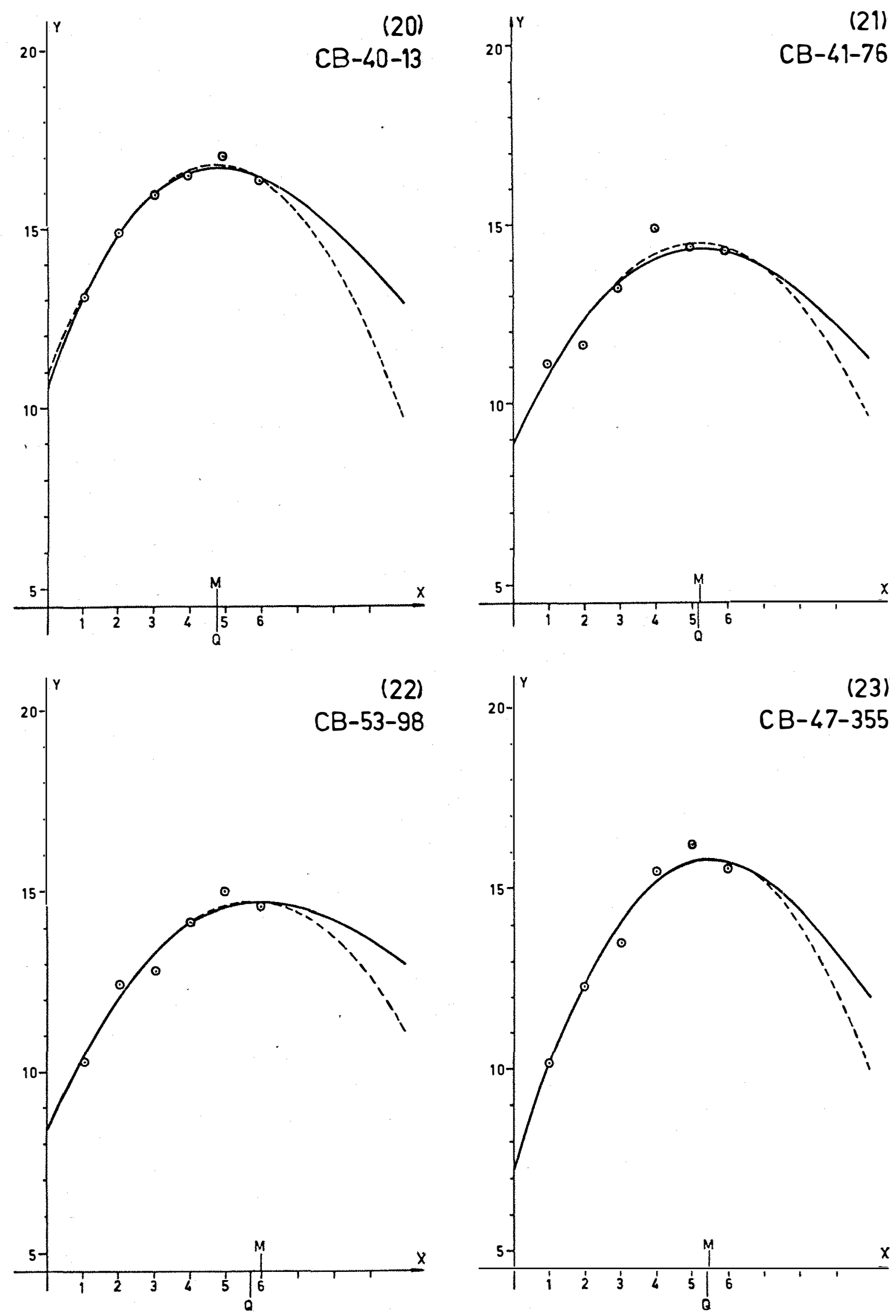
55.
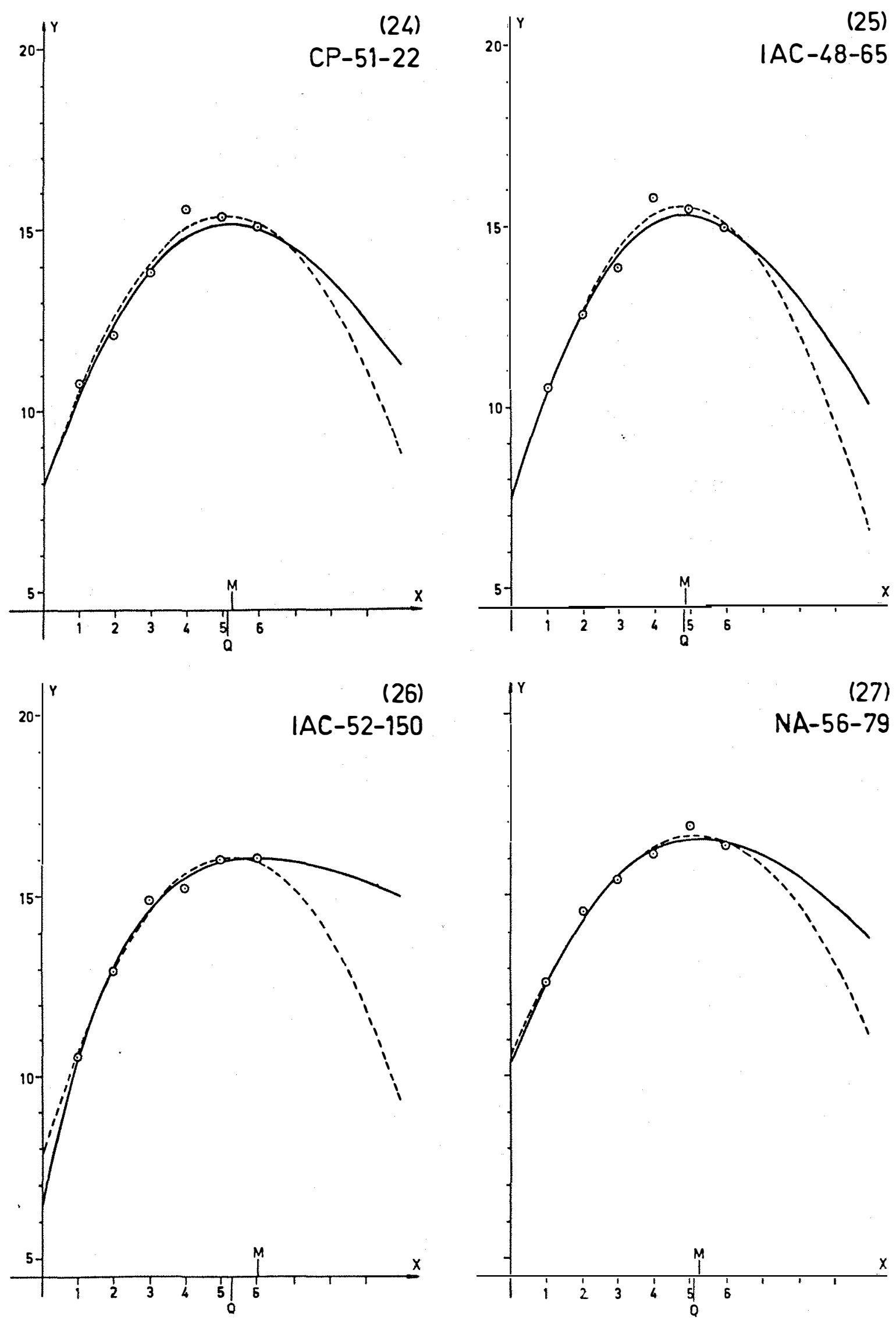
Devemos acrescentar que o método de Marquardt foi iniciado, para todas as variedades, com $b_{11}=500$ e sempre houve convergência, lenta quando $b_{1}$ tem valor pequeno, para resultados semelhantes aos obtidos.

Quanto aos métodos de Gauss e de Newton,a apli cação do procedimento anterior, para o início do processo iterativo, dará uma matriz Hessiana, ou sua aproximação, sin gular. Uma opção foi selecionar um conjunto de valores para cada parâmetro e adotar aquela combinação de valores, dentre todas as possíveis, que produzisse a menor soma de quadrados dos desvios. A forma da superfície destas impôs a seleção de muitos valores e, mesmo assim, em alguns casos o processo in ciou-se longe do mínimo, com uma consequente demora na convergência. Além disso, a matriz Hessiana nem sempre é defini da positiva, devido à acumulação de erros de arredondamento, o que exigiu que o método de Newton também fosse modificado para garantir a convergência. Considerando essas dificuldades e que, por qualquer dos métodos, os resultados foram próximos, indicamos o método de Marquardt como o mais eficiente.

\subsection{Análise dos Ajustamentos}

Já é possível uma discussão dos resultados, levando-se em conta, principalmente, a propriedade da função de Mitscherlich, a qual motivou este trabalho, de poder apre 
sentar um decrescimențo menos intenso após o máximo. Em primeiro lugar, destaca-se a variedade de número 7 (CB-56-126) cujos dados levaram a um ajustamento pela segunda Lei de Mitscherlich que não indica um máximo, pois temos $\hat{b}_{4}<0$. Aí, o último ponto ainda apresenta um valor alto de Pol\% da cana e, desde que fosse a média de algumas repetições, deveriaser mais bem analisado. Se, entretanto, rejeitarmos a hipótese de que os dados tenham sido obtidos com precisão insuficiente, o comportamento da Segunda Lei indicaria que o período de ex perimentação foi curto. Não se tem, com segurança, a época em que a porcentagem de Pol começa a decrescer. As outras 26 va riedades podem ser divididas em dois grupos. Um, que abrange aquelas para as quais a Segunda Lei, ao apresentar $\hat{b}_{4}$ positi vo e mais próximo de zero, indica un decréscimo pouco intenso de Pol\% da cana depois que o máximo foi atingido, aliás, acom panhando a tendência dos dados experimentais. Enquadram-se aí as variedades de números $2,3,4,5,10,19$ e 26 , ou seja, CB-40-77, $\mathrm{CB}-41-76, \mathrm{CB}-46-47, \mathrm{CB}-49-260, \mathrm{CO}-740, \mathrm{IAC}-80-326$ e IAC-52-150. Outro, que compreende aquelas para as quais os dois modelos descrevem de forma semelhante o fenômeno de maturação, mas nas quais, ainda assim, a Segunda Lei é mais confiável para extrapolação. Enquanto que para as variedades do primeiro gru po a soma de quadrados dos desvios obtida pelo ajustamento da Lei de Mitscherlich foi sempre menor do que a obtida pelo ajustamento da equação Quadrática, no segundo grupo, se não foi menor, ficou próxima. Percebem-se maiores dificuldades, 
devido ao período mais curto de experimentação, no estudo das variedades de 20 a 27 onde, independentemente do que os mode los possam indicar, a ausência de outros pontos coloca em dú vida se a tendência é de um decrescimento rápido ou lento. Quanto à época de máxima Pol\% da cana, a Lei de Mitscherlich indica o início de outubro para a variedade 26 (IAC-52-150) enquanto que o modelo quadrático indica cêrca de três semanas antes. Mas, para as outras variedades, esta diferença nunca foi superior a duas semanas, sendo de apenas alguns dias na maioria dos casos. Um período de experimentação curto pode trazer outras consequências graves. Assim, se, por exemplo, a porcentagem de Pol para a variedade de número 5 ( $\mathrm{CB}-49-260)$ tivesse sido pesquisada até o sexto mês apenas, teriamos uma indicação de máximo, aproximadamente, dois meses antes do obtido.

Para uma análise por critérios estatísticos, a Tabela 5 expõe, usando as definições da seção 3.2 .3 , e considerando somente os valores médios: o Coeficiente deVariação, o Coeficiente de Determinação e os valores do Teste F para a regressão Quadrática e para a regressão pela Segunda Lei de Mitscherlich, à qual atribuimos 3 graus de liberdade. Uma regressão significativa ao nível de 5\% indicou-se por um asterisco e por dois asteriscos a significação ao nível de 1\%. A comparação, em cada variedade, dos Coeficientes de Variação e dos Coeficientes ajustados de Determinação, obtidos pelo ajustamento dos dois modelos, não permitiu decidir se um de- 
les é mais adequado, por terem sido sempre próximos os resul tados. Entretanto, esses coeficiertes se afastaram dos teorí camente ideais, o (zero) para o primeiro e 1 (um) para o outro, principalmente o de Variação, nas variedades que apresen taram pontos intermediários de Pol\% da cana excessivamente discrepantes dos demais $(2 ; 6$ e 11$)$, motivando suspeitas sobre tais dados. Por outro lado, nos casos em que as duas cur vas praticamente coincidem, o valor de $\mathrm{F}$ para a regressão Quadrática ficou um tanto acima do $F$ para a Segunda Lei de Mitscherlich. Como, para as variedades de 20 a 27 , os dados são médias de três repetições, cujos valores são conhecidos e estão expostos na Tabela 7 do apêndice, é possível determi. nar uma outra estimativa, mais correta, do erro experimental. A análise foi, então, modificada, conforme a discussão na seção 3.2.3., e os resultados obtidos estão na Tabela 8 do apêndice. As conclusões anteriores, entretanto, não se alteraram, especialmente quanto a discrepância entre os valores de $F$ quando as duas curvas quase coincidem. Esses resultados ressaltam que a eleição entre o modelo linear e o não linear por critérios exclusivamente estatísticos, próprios para o primeiro modelo, não é correta. 
60.

Tabela 5. Coeficientes de Variação (C.V.), Coeficientes ajustados de Determinação $\left(\overline{\mathrm{R}}^{2}\right)$ e valores do Teste $\mathrm{F}$ para a Segun da Lei deMitscherlich e para a Regressão Quadrática.

\begin{tabular}{|c|c|c|c|c|c|c|c|}
\hline \multirow{2}{*}{ No } & \multirow{2}{*}{ Variedade } & \multicolumn{3}{|c|}{ Mitscherlich } & \multicolumn{3}{|c|}{ Quadrático } \\
\hline & & C.V. $(\%)$ & $\overline{\mathrm{R}}^{2}$ & $\mathrm{~F}$ & C.V. $(\%)$ & $\overline{\mathrm{R}}^{2}$ & $\mathbf{F}$ \\
\hline 1 & $C B-40-13$ & 4,34 & 0,7299 & $7,31^{*}$ & 3,78 & 0,7949 & $14,56^{* *}$ \\
\hline 2 & $\mathrm{CB}-40-77$ & 12,02 & 0,6878 & 6,14 & 11,74 & 0,7018 & $9,24^{*}$ \\
\hline 3 & $C B-41-76$ & 4,15 & 0,9465 & $42,30^{* *}$ & 5,73 & 0,8980 & $31,83^{* *}$ \\
\hline 4 & $C B-46-47$ & 3,37 & 0,9289 & $31,47^{* *}$ & 4,18 & 0,8906 & $29,48^{* *}$ \\
\hline 5 & $C B-49-260$ & 4,71 & 0,9010 & $22,23^{* *}$ & 4,85 & 0,8951 & $30,88^{* *}$ \\
\hline 6 & $\mathrm{CB}-56-121$ & 8,57 & 0,4364 & 2,81 & 7,57 & 0,5593 & 5,44 \\
\hline 7 & CB-56-126 & 2,86 & 0,9524 & $47,70^{* *}$ & 4,15 & 0,9001 & $32,53^{* *}$ \\
\hline 8 & $C B-56-164$ & 2,86 & 0,8847 & $18,90^{* *}$ & 2,81 & 0,8888 & $28,96^{* *}$ \\
\hline 9 & C0-178 & 4,70 & 0,9132 & $25,54^{* *}$ & 4,22 & 0,9301 & $47,55^{* *}$ \\
\hline 10 & $\mathrm{CO}-740$ & 1,54 & 0,9940 & $386,99^{* *}$ & 3,85 & 0,9624 & $90,48^{* *}$ \\
\hline 11 & $\mathrm{C} 0-775$ & 8,73 & 0,7984 & $10,24^{*}$ & 8,12 & 0,8253 & $17,53^{* *}$ \\
\hline 12 & IAC $-48-31$ & 4,77 & 0,9000 & $21,99^{* *}$ & 5,55 & 0,8643 & $23,29^{* *}$ \\
\hline 13 & IAC $-51-201$ & 4,72 & 0,9303 & $32,12^{* *}$ & 5,28 & 0,9127 & $35,57^{* *}$ \\
\hline 14 & IAC $-51-264$ & 4,38 & 0,9368 & $35,61^{* *}$ & 4,43 & 0,9354 & $51,66^{* *}$ \\
\hline 15 & IAC-51-285 & 4,93 & 0,9166 & $26,63^{* *}$ & 5,37 & 0,9008 & $32,79^{* *}$ \\
\hline 16 & IAC-52-130 & 3,51 & 0,9328 & $33,38^{* *}$ & 3,65 & 0,9273 & $45,65^{* *}$ \\
\hline 17 & IAC-52-172 & 2,58 & 0,9852 & $156,34^{* *}$ & 2,10 & 0,9902 & $354,80^{* *}$ \\
\hline 18 & IAC $-52-179$ & 4,91 & 0,9364 & $35,33^{* *}$ & 3,84 & 0,9611 & $87,37^{* *}$ \\
\hline 19 & IAC $-80-326$ & 3,01 & 0,9732 & $85,75^{* *}$ & 4,09 & 0,9504 & $68,09^{* *}$ \\
\hline 20 & $C B-40-13$ & 1,43 & 0,9751 & $66,17^{*}$ & 1,02 & 0,9873 & $194,75^{* *}$ \\
\hline 21 & $C B-41-76$ & 6,32 & 0,7125 & 5,13 & 4,97 & 0,8224 & $12,58^{*}$ \\
\hline 22 & $\mathrm{CB}-53-98$ & 4,18 & 0,8999 & 15,99 & 3,34 & 0,9364 & $37,82^{* *}$ \\
\hline 23 & $C B-47-355$ & 4,09 & 0,9396 & $26,93^{*}$ & 3,23 & 0,9625 & $65,13^{* *}$ \\
\hline 24 & $\mathrm{CP}-51-22$ & 4,91 & 0,8773 & 12,91 & 3,40 & 0,9412 & $41,04^{* *}$ \\
\hline 25 & IAC $-48-65$ & 4,52 & 0,8995 & 15,91 & 3,00 & 0,9557 & $54,92^{* *}$ \\
\hline 26 & IAC-52-150 & 2,06 & 0,9809 & $86,74^{*}$ & 2,18 & 0,9788 & $116,24^{* *}$ \\
\hline 27 & NA-56-79 & 2,19 & 0,9533 & $35,06^{*}$ & 1,72 & $0,971.3$ & $85,53^{* *}$ \\
\hline
\end{tabular}


61.

5. CONCLUSÕES

Dos métodos do gradiente estudados, para a es timação dos parâmetros da Segunda Lei de Mitscherlich, o de Marquardt teve melhor desempenho. Foi proposto, e empregado, um algoritmo que, incorporado ao método de Marquardt, acelerou a convergência do processo e eliminou a necessidade de uma pesquisa prévia de valores iniciais. Mais ainda, após pou cás iterações, sempre ficou definida a ordem de grandeza tan to de $b_{2}$ como de $b_{4}$ e se $b_{1}$ aumentaria ou não, isto é, se a Lei de Mitscherlich apresentaria um comportamento equivalente ao da parábola ou se teria um decrescimento menos intenso após o máximo. Concluiu-se, também, que os problemas de convergência estão relacionados com a forma da superfície das somas de quadrados, estreita e alongada na direção do eixo $b_{1}$. Uma análise visual dos gráficos obtidos evidenciou que os ajustamentos se assemelham, por um modelo ou 
outro, até o máximo, mas, daí em diante, a Segunda Lei de Mitscherlich acompanha melhor a tendência dos dados, tornandose mais confiável a extrapolação. Entretanto, mesmo para a Se gunda Lei, esta confiabilidade diminuiu para as variedades de 20 a 27, pois, o máximo ocorreu no final do período experimental e não sobraram pontos suficientes para caracterizar o fenômeno a partir dar. Conclui-se, então, que a Lei de Mitscherlich dará uma visão mais informativa desde que o período experimental seja suficientemente longo, como para as variedades de 1 a 19. Na verdade, tudo indica que também o intervalo de um mês, entre uma medição e outra, deva ser diminuido. Quanto à análise estatística, Coeficientes de Variação acima de 6\%, por qualquer dos modelos, ocorreram pa ra as variedades com dados intermediários discrepantes. Por outro lado, os valores do Coeficiente de Determinação, corri gido ou não, e do Teste $F$ não foram suficientes para indicar o melhor ajustamento. 
63.

6. LITERATURA CITADA

BARD, Y., 1970. Comparison of Gradient Methods for the Solution of Nonlinear Problems. SIAM J. Num. Anal. 7: 157-186.

BARD, Y., 1974. Nonlinear Parameter Estimation. Nova York, Aca demic Press Inc. $341 \mathrm{p}$.

BAYMA, A.C., 1975. Manual de Técnicas de Laboratório e Fabricação de Açúcar de Cana. Rio de Janeiro, Instituto de Açú car e do Alcool, p. 92-109, Coleção Canavieira no 18.

DAVIDON, W.C., 1959. Variable Metric Method for Minimization. Atomic Energy Commission Research and Development Report no 5990, Argonne National Laboratories.

DRAPER, N.R. e H. SMITH, 1981. Applied Regression Analysis. 2. edição, Nova York, John Wiley. 710 p.

FLETCHER, R. e M.J.D. POWELL, 1963. A Rapidly Convergent Descent Method for Minimization. The Computer Journal. 6: 163-168. 
FLETCHER, R., 1970. A New Approach to Variable Metric Algorithms. The Computer Journal. 13: 317-322.

GUTTMAN, I. e D.A. MEETER, 1965. On Beale's Measures of Nonlinearity. Technometrics. $7: 623-637$.

HARTLEY, H.0., 1961. The Modified Gauss-Newton Method for the Fitting of Nonlinear Regression Functions by Least Squares. Technometrics. 3 : 269-280.

HARTLEY, H.O., 1964. Exact Confidence Regions for the Parameters in Nonlinear Regression Law. Biometrika. 51: 347-353.

HOFFMANN, R. e S. VIEIRA, 1977. Análise de Regressão. São Paulo, Ed. da Universidade de São Paulo. 339 p.

KITRELL, J.R., R. MEZAKI e C.C.WATSON, 1965. Estimation of Parameters for Nonlinear Least Squares Analysis. Industrial. and Engineering Chemistry. 57: 18-27.

LEVENBERG, K., 1944. A Method for the Solution of Certain Nonlinear Problems in Least Squares. Quart. Appl. Math. 2: 164-168.

MARQUARDT, D.W., 1963. An Algorithm for Least Squares Estimation of Nonlinear Parameters. SIAM J. Num. Analysis. 11: 431-441.

PIMENTEL GOMES, F., 195̆l. A Interpolação da Lei de Mitscherlich e a Análiseda Variância em Experimentos de Adubação. Anais da E.S.A. "Luiz de Queiroz". 8: 185-204.

PIMENTEL GOMES, F., 1953. The Use of Mitscherlich Law in the Ana lysis of Experiments with Fertilizers. Biometrics. 9: 498-517.

PIMENTEL GOMES, F., 1969. Novos Aspectos do Estudo Econômico 
de Adubação. Fertilité. 34: 3-9.

PIMENTEL GOMES, F., 1978. A Lei de Mitscherlich Aplicada a En saios de Adubação. Anais da Reunião Brasileira da Socieda de Internacional de Biometria na Comemoração do 90 Aniversário do Instituto Agronômico de Campinas.

PIMENTEL GOMES, F. e H. CAMPOS, 1966. Resultados de Ensaios de Adubação. In: Cultura e Adubação do Milho. Instituto Bra sileiro da Potassa. Brasil.

PIMENTEL GOMES, F. e E. MALAVOLTA, 1949. Aspectos Matemáticos e Estatísticos da Lei de Mitscherlich. Anais da E.S.A."Luiz de Queiroz". 6: 193-229.

PIMENTEL GOMES, F. e I.R. NOGUEIRA, 1975. Extrapolação ou Projeção: Uma técnica Difícil e Perigosa. Ciência e Cultura. 29: $1386-1389$.

SEAL, H.L., 1967. The Historical Development of the Gauss Li near Model. Biometrika. 54: 1-24.

SMITH, F.B. e D.F. SHANNO, 1971. An Improved Marquardt Procedure for Nonlinear Regressions. Technometrics. 13: 63-74.

SILVA, MariaA.P., 1978. Segunda Aproximação de Mitscherlich, $\mathrm{Y}=\mathrm{A} \cdot 1-10^{-\mathrm{c}(\mathrm{x}+\mathrm{b})} \cdot 10^{-\mathrm{k}(\mathrm{x}+\mathrm{b})^{2}}$, Aplicada à Adubação Mineral. Piracicaba, ESALQ/USP, 96 p. (Dissertação de Mestrado).

SILVA, Maria A.P., 1980. Métodos de Estimação dos Parâmetros do Modelo $\mathrm{Y}=\mathrm{A}\left(1-10^{-\mathrm{cx}}\right) 10^{-\mathrm{kx}}+\mathrm{e}$. Aplicação a Dados de Maturação da Cana-de-açúcar. Piracicaba, ESALQ/USP, 85 p. (Tese de Doutoramento). 
UD0, Margareth C.T., 1983. Estudo de Funções que se Ajustam à Maturação da Cana-de-Açúcar. Piracicaba, ESALQ/USP, 74 p. (Dissertação de Mestrado).

ZAGATT0, A.G. e F. PIMENTEL GOMES, 1967. Aspectos Econômicos da Adubação. Em ${ }^{2}$ MaLAVolta, E., Manual de Química Agrícola. Adubos e Adubações. 2. Ed. Biblioteca Agronômica "Ceres". 
67.

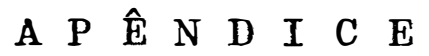


68.

Tabela 6. Estimativas dos parâmetros da Segunda Lei de Mitscherlich obtidas pelo método de Marquardt finalizado

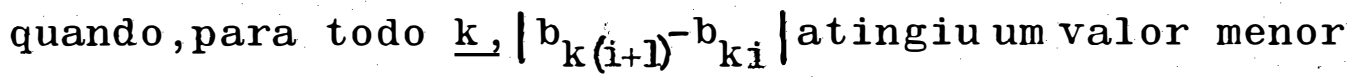
do que (1) $10^{-2} \mathrm{~L}$, (2) $10^{-3} \mathrm{~L}$ e (3) $10^{-4} \mathrm{~L}$ com $\mathrm{L}=\mathrm{b}_{\mathrm{k}, \mathrm{i}}+10^{-3}$.

\begin{tabular}{|c|c|c|c|c|c|c|c|}
\hline $\begin{array}{l}\text { No } \\
\text { Variedade }\end{array}$ & & Iter. & $\hat{b}_{1}$ & $\hat{\mathrm{b}}_{2}$ & $\hat{\mathrm{b}}_{3}$ & $\hat{\mathrm{b}}_{4}$ & SQDesvios \\
\hline 1 & (1) & 8 & 32,6154 & 3,4547 & 0,057974 & 0,001469 & 1,9891 \\
\hline \multirow[t]{2}{*}{$C B-40-13$} & (2) & 287 & 237,0380 & 4,4091 & 0,005540 & 0,002136 & 1,8870 \\
\hline & (3) & 324 & 295,6750 & 4,4325 & 0,004402 & 0,002153 & 1,8856 \\
\hline \multirow[t]{2}{*}{$\begin{array}{l}2 \\
\mathrm{CB}-40-77\end{array}$} & $\begin{array}{l}(1) \\
(2)\end{array}$ & 8 & 18,4890 & 0,1217 & 0,240052 & 0,000747 & 12,6864 \\
\hline & (3) & 11 & 18,4875 & 0,1215 & 0,240120 & 0,000746 & 12,6863 \\
\hline $\begin{array}{l}3 \\
\mathrm{CB}-41-76\end{array}$ & $\begin{array}{l}(1) \\
(2) \\
(3)\end{array}$ & 7 & 18,5599 & 0,1573 & 0,252979 & 0,000918 & 1,5364 \\
\hline $\begin{array}{l}4 \\
\mathrm{CB}-46-77\end{array}$ & $\begin{array}{l}(1) \\
(2) \\
(3)\end{array}$ & 6 & 19,6116 & 0,6359 & 0,241455 & 0,000933 & 1,1797 \\
\hline $\begin{array}{l}5 \\
C B-49-260\end{array}$ & $\begin{array}{l}(1) \\
(2) \\
(3)\end{array}$ & 6 & 19,1377 & 0,8190 & 0,190927 & 0,000771 & 2,0488 \\
\hline \multirow{2}{*}{$\begin{array}{l}6 \\
\mathrm{CB}-56-121\end{array}$} & (1) & 42 & 73,6452 & 3,8563 & 0,018024 & 0,001909 & 6,5593 \\
\hline & $\begin{array}{l}(2) \\
(3)\end{array}$ & 277 & 329,9340 & 4,1172 & 0,003623 & 0,002135 & 6,5137 \\
\hline $\begin{array}{l}7 \\
\mathrm{CB}-56-126\end{array}$ & $\begin{array}{l}\text { (1) } \\
\text { (2) } \\
\text { (3) }\end{array}$ & 7 & 16,6087 & 0,3824 & 0,337209 & $-0,000023$ & 0,7702 \\
\hline \multirow{2}{*}{$\begin{array}{l}8 \\
\mathrm{CB}-56-164\end{array}$} & (1) & 6 & 21,3923 & 2,5092 & 0,117660 & 0,000970 & 0,7793 \\
\hline & $\begin{array}{l}(2) \\
(3)\end{array}$ & 8 & 21,4052 & 2,5115 & 0,117485 & 0,000971 & 0,7793 \\
\hline \multirow{2}{*}{$\begin{array}{c}9 \\
\mathrm{CO}-178\end{array}$} & (1) & 46 & 65,3354 & 1,7851 & 0,026939 & 0,003116 & 1,8071 \\
\hline & $\begin{array}{l}(2) \\
(3)\end{array}$ & 259 & 260,0910 & 1,9868 & 0,006034 & 0,003546 & 1,7754 \\
\hline
\end{tabular}


Tabela 6 . Continuação

\begin{tabular}{|c|c|c|c|c|c|c|c|}
\hline $\begin{array}{l}\text { № } \\
\text { Vari edade }\end{array}$ & & Iter. & $\hat{b}_{1}$ & $\hat{b}_{2}$ & $\hat{b}_{3}$ & $\hat{\mathrm{b}}_{4}$ & QDesvios \\
\hline $\begin{array}{l}10 \\
\mathrm{CO}-740\end{array}$ & $\begin{array}{l}(1) \\
(2) \\
(3)\end{array}$ & 7 & 19,4308 & 0,1763 & 0,232098 & 0,000545 & 0,2347 \\
\hline $\begin{array}{l}11 \\
\mathrm{CO}-775\end{array}$ & $\begin{array}{l}\text { (1) } \\
\text { (2) } \\
(3)\end{array}$ & $\begin{array}{r}68 \\
217\end{array}$ & $\begin{array}{l}168,1490 \\
499,1740\end{array}$ & $\begin{array}{l}1,0550 \\
1,1148\end{array}$ & $\begin{array}{l}0,014057 \\
0,004532\end{array}$ & $\begin{array}{l}0,005215 \\
0,005461\end{array}$ & $\begin{array}{l}8,2875 \\
8,2317\end{array}$ \\
\hline $\begin{array}{l}12 \\
\text { IAC-48-31 }\end{array}$ & $\begin{array}{l}(1) \\
(2) \\
(3)\end{array}$ & 6 & 26,1400 & 0,6593 & 0,141309 & 0,003251 & 2,1212 \\
\hline $\begin{array}{l}13 \\
\text { IAC-51-121 }\end{array}$ & $\begin{array}{l}\text { (1) } \\
\text { (2) } \\
\text { (3) }\end{array}$ & $\begin{array}{l}6 \\
7\end{array}$ & $\begin{array}{l}24,7750 \\
24,7811\end{array}$ & $\begin{array}{l}0,6158 \\
0,6161\end{array}$ & $\begin{array}{l}0,118399 \\
0,118346\end{array}$ & $\begin{array}{l}0,002751 \\
0,002752\end{array}$ & $\begin{array}{l}1,7208 \\
1,7208\end{array}$ \\
\hline 14 & (1) & 31 & 52,0185 & 1,0818 & 0,045378 & 0,003950 & 1,7813 \\
\hline IAC $-5 I-264$ & $\begin{array}{l}(2) \\
(3)\end{array}$ & $\begin{array}{l}87 \\
91\end{array}$ & $\begin{array}{l}76,3932 \\
76,9991\end{array}$ & $\begin{array}{l}1,1966 \\
1,1985\end{array}$ & $\begin{array}{l}0,028600 \\
0,028340\end{array}$ & $\begin{array}{l}0,004299 \\
0,004305\end{array}$ & $\begin{array}{l}1,7734 \\
1,7733\end{array}$ \\
\hline $\begin{array}{l}15 \\
\text { IAC-5I-285 }\end{array}$ & $\begin{array}{l}(1) \\
(2) \\
(3)\end{array}$ & $\begin{array}{l}6 \\
8\end{array}$ & $\begin{array}{l}31,0080 \\
31,0034\end{array}$ & $\begin{array}{l}0,7556 \\
0,7554\end{array}$ & $\begin{array}{l}0,095465 \\
0,095488\end{array}$ & $\begin{array}{l}0,003407 \\
0,003407\end{array}$ & $\begin{array}{l}2,1741 \\
2,1741\end{array}$ \\
\hline $\begin{array}{l}16 \\
\text { IAC-52-130 }\end{array}$ & $\begin{array}{l}(1) \\
(2) \\
(3)\end{array}$ & $\begin{array}{l}7 \\
8\end{array}$ & $\begin{array}{l}47,3000 \\
47,3389\end{array}$ & $\begin{array}{l}1,5276 \\
1,5281\end{array}$ & $\begin{array}{l}0,048929 \\
0,048879\end{array}$ & $\begin{array}{l}0,003695 \\
0,003696\end{array}$ & $\begin{array}{l}1,1412 \\
1,1412\end{array}$ \\
\hline 17 & (1) & 51 & 67,6274 & 1,1062 & 0,026706 & 0,003447 & 0,4723 \\
\hline IAC $-52-172$ & $\begin{array}{l}(2) \\
(3)\end{array}$ & $\begin{array}{l}209 \\
210\end{array}$ & $\begin{array}{l}213,0500 \\
213,0640\end{array}$ & $\begin{array}{l}1,2441 \\
1,2441\end{array}$ & $\begin{array}{l}0,007721 \\
0,007720\end{array}$ & $\begin{array}{l}0,003902 \\
0,003902\end{array}$ & $\begin{array}{l}0,4519 \\
0,4519\end{array}$ \\
\hline 18 & (1) & 72 & 157,6620 & 1,2219 & 0,011450 & 0,004358 & 1,7178 \\
\hline IAC $-52-179$ & $\begin{array}{l}(2) \\
(3)\end{array}$ & $\begin{array}{l}249 \\
252\end{array}$ & $\begin{array}{l}618,7460 \\
623,7630\end{array}$ & $\begin{array}{l}1,2828 \\
1,2830\end{array}$ & $\begin{array}{l}0,002797 \\
0,002774\end{array}$ & $\begin{array}{l}0,004577 \\
0,004578\end{array}$ & $\begin{array}{l}1,6722 \\
1,6722\end{array}$ \\
\hline $\begin{array}{l}19 \\
\text { IAC-80-326 }\end{array}$ & $\begin{array}{l}(1) \\
(2) \\
(3)\end{array}$ & 6 & 18,7998 & 0,3635 & 0,213554 & 0,000683 & 0,8118 \\
\hline
\end{tabular}


Tabela 6 . Continuação

\begin{tabular}{|c|c|c|c|c|c|c|c|}
\hline $\begin{array}{l}\text { No } \\
\text { Vari edade }\end{array}$ & & Iter. & $\hat{\mathrm{b}}_{1}$ & $\hat{\mathrm{b}}_{2}$ & $\hat{\mathrm{b}}_{3}$ & $\hat{\mathrm{b}}_{4}$ & SQDesvios \\
\hline 20 & (1) & 16 & 28,7530 & 2,6474 & 0,077912 & 0,001886 & 0,0990 \\
\hline \multirow[t]{2}{*}{$C B-40-13$} & (2) & 149 & 63,6393 & 3,2675 & 0,026552 & 0,002626 & 0,0846 \\
\hline & (3) & 229 & 78,9178 & 3,3454 & 0,020664 & 0,002726 & 0,0842 \\
\hline 21 & (1) & 12 & 40,6919 & 3,1261 & 0,034089 & 0,002045 & 2,1465 \\
\hline \multirow[t]{2}{*}{ CB-41-76 } & (2) & 189 & 195,1240 & 3,4149 & 0,006286 & 0,002688 & 1,3130 \\
\hline & (3) & 249 & 257,3460 & 3,4341 & 0,004722 & 0,002721 & 1,3109 \\
\hline 22 & (1) & 14 & 26,0169 & 2,5694 & 0,065146 & 0,001451 & 0,6122 \\
\hline \multirow[t]{2}{*}{ CB-53-98 } & (2) & 147 & 53,1355 & 3,0381 & 0,025556 & 0,002105 & 0,5961 \\
\hline & (3) & 221 & 76,6887 & 3,1504 & 0,016784 & 0,002269 & 0,5951 \\
\hline 23 & (1) & 56 & 72,9124 & 2,0903 & 0,022430 & 0,003005 & 0,6434 \\
\hline \multirow[t]{2}{*}{$\mathrm{CB}-47-355$} & (2) & 279 & 330,6800 & 2,2428 & 0,004509 & 0,003441 & 0,6181 \\
\hline & (3) & 328 & 397,7290 & 2,2495 & 0,003734 & 0,003461 & 0,6170 \\
\hline 24 & (1) & 58 & 74,3495 & 2,4005 & 0,021368 & 0,003085 & 0,7000 \\
\hline$C P-51-22$ & $\begin{array}{l}(2) \\
\text { (3) }\end{array}$ & 277 & 300,8070 & $-2,5545$ & 0,004835 & 0,003467 & 0,6756 \\
\hline 25 & (1) & 54 & 74,3777 & 2,0135 & 0,023855 & 0,003776 & 0,5838 \\
\hline IAC $-48-65$ & (2) & 264 & 326,8740 & 2,1652 & 0,004943 & 0,004235 & 0,5588 \\
\hline $\begin{array}{l}26 \\
\text { IAC-52-150 }\end{array}$ & $\begin{array}{l}\text { (1) } \\
\text { (2) } \\
\text { (3) }\end{array}$ & 6 & 17,9106 & 0,9655 & 0,198361 & 0,000610 & 0,1739 \\
\hline 27 & (1) & 16 & 26,9168 & 2,7022 & 0,079488 & 0,001519 & 0,2243 \\
\hline \multirow[t]{2}{*}{ NA-56-79 } & (2) & 141 & 49,6351 & 3,2819 & 0,033264 & 0,002160 & 0,2171 \\
\hline & (3) & 158 & 51,4633 & 3,3018 & 0,031798 & 0,002184 & 0,2171 \\
\hline
\end{tabular}


71.

Tabela 7. Resultados de Pol\% da cana, obtidos pela Usina São João, Araras (S.P), no ano de 1979 .

\begin{tabular}{lcccccccc}
\hline No & & \multicolumn{7}{c}{ Meses } \\
\cline { 2 - 8 } & Amoriedade & Amostra & $\begin{array}{c}1 \\
\text { maio }\end{array}$ & $\begin{array}{c}2 \\
\text { junho }\end{array}$ & $\begin{array}{c}3 \\
\text { julho }\end{array}$ & $\begin{array}{c}4 \\
\text { agos. }\end{array}$ & $\begin{array}{c}5 \\
\text { set. }\end{array}$ & out. \\
\hline 20 & 1 & 12,59 & 14,62 & 15,74 & 16,15 & 17,04 & 16,23 \\
CB-40-13 & 2 & 13,46 & 15,00 & 16,26 & 16,80 & 16,39 & 16,16 \\
& 3 & 13,17 & 15,09 & 15,75 & 16,41 & 17,34 & 16,49 \\
& Média & 13,07 & 14,90 & 15,92 & 16,45 & 16,92 & 16,29
\end{tabular}

\begin{tabular}{lcrrrrrr}
\hline 21 & 1 & 13,04 & 11,54 & 13,33 & 14,83 & 14,78 & 14,35 \\
$\mathrm{CB}-41-76$ & 2 & 10,54 & 10,97 & 12,96 & 14,45 & 13,98 & 14,14 \\
& 3 & 9,76 & 12,26 & 13,32 & 15,44 & 14,21 & 14,13 \\
\hline & Média & 11,11 & 11,59 & 13,20 & 14,91 & 14,32 & 14,21
\end{tabular}

\begin{tabular}{lcrccccc}
\hline 22 & 1 & 10,35 & 12,31 & 13,10 & 14,32 & 15,62 & 15,18 \\
CB-53-98 & 2 & 9,90 & 12,79 & 13,09 & 14,36 & 14,70 & 14,58 \\
& 3 & 10,58 & 12,35 & 12,24 & 13,86 & 14,64 & 14,01 \\
\hline & Média & 10,28 & 12,48 & 12,81 & 14,18 & 14,99 & 14,59
\end{tabular}

\begin{tabular}{lcrccccc}
\hline 23 & 1 & 10,15 & 12,23 & 13,37 & 15,45 & 16,87 & 15,28 \\
$\mathrm{CB}-47-355$ & 2 & 9,95 & 12,52 & 13,52 & 15,69 & 15,86 & 15,52 \\
& 3 & 10,51 & 12,36 & 13,82 & 15,36 & 15,96 & 15,82 \\
\hline & Média & 10,20 & 12,37 & 13,57 & 15,50 & 16,23 & 15,54
\end{tabular}


Tabela 7. Continuação

\begin{tabular}{lcccccccc}
\hline \multirow{2}{*}{$\begin{array}{l}\text { Vo } \\
\text { Variedade }\end{array}$} & Amostra & & $\begin{array}{c}\text { Meses } \\
\text { maio }\end{array}$ & $\begin{array}{c}2 \\
\text { junho }\end{array}$ & $\begin{array}{c}3 \\
\text { julho }\end{array}$ & $\begin{array}{c}4 \\
\text { agos. }\end{array}$ & $\begin{array}{c}5 \\
\text { set. }\end{array}$ & $\begin{array}{c}6 \\
\text { out. }\end{array}$ \\
\hline 24 & 1 & 10,66 & 11,92 & 13,58 & 15,37 & 13,92 & 15,43 \\
CP-51-22 & 2 & 10,77 & 12,04 & 13,86 & 16,21 & 16,77 & 15,14 \\
& 3 & 11,09 & 12,52 & 14,17 & 15,19 & 15,41 & 14,71 \\
\hline & Média & 10,84 & 12,16 & 13,87 & 15,59 & 15,37 & 15,09
\end{tabular}

\begin{tabular}{lccccccc}
\hline 25 & 1 & 10,66 & 12,56 & 13,65 & 15,69 & 15,54 & 14,55 \\
IAC-48-65 & 2 & 10,49 & 12,96 & 14,30 & 16,05 & 15,30 & 14,94 \\
& 3 & 10,79 & 12,47 & 13,83 & 15,71 & 15,87 & 15,45 \\
\hline & Média & 10,65 & 12,66 & 13,93 & 15,82 & 15,57 & 14,98
\end{tabular}

\begin{tabular}{lrrrrrrr}
\hline 26 & 1 & 9,62 & 13,10 & 15,57 & 15,60 & 15,85 & 15,75 \\
IAC-52-150 & 2 & 11,05 & 12,83 & 14,42 & 15,18 & 16,17 & 15,83 \\
& 3 & 11,07 & 13,05 & 14,80 & 14,97 & 16,08 & 16,48 \\
\hline & Média & 10,58 & 12,99 & 14,93 & 15,25 & 16,03 & 16,02
\end{tabular}

\begin{tabular}{llllllll}
\hline 27 & 1 & 11,94 & 14,37 & 15,84 & 16,06 & 16,48 & 16,54 \\
NA-56-79 & 2 & 12,96 & 14,60 & 14,79 & 16,05 & 17,23 & 15,86 \\
& 3 & 12,94 & 14,78 & 15,62 & 16,26 & 16,96 & 16,60 \\
\hline & Média & 12,61 & 14,58 & 15,42 & 16,12 & 16,89 & 16,33
\end{tabular}


Tabela 8. Valores do Teste $\mathrm{F}$ para a Regressão e para os Desvios da Regressão; Coeficientes de Variação (C.V.) e Coeficientes de Determinação $\left(R^{2}\right)$ para as variedades de 20 a 27 , com três repetições em cada época, de acordo com os modelos: Mitscherlich e Quadrático.

\begin{tabular}{|c|c|c|c|c|c|}
\hline $\begin{array}{l}\text { No } \\
\text { Variedade }\end{array}$ & Modelo & $\begin{array}{c}\text { F } \\
\text { Regr. }\end{array}$ & $\begin{array}{c}F \\
\text { Desv. Regr. }\end{array}$ & C.V. $(\%)$ & $\mathrm{R}^{2}$ \\
\hline 20 & Mitscherlich & $81,94^{* *}$ & 1,26 & 2,22 & 0,9442 \\
\hline $\mathrm{CB}-40-13$ & Quadrático & $123,21^{* *}$ & 0,64 & 2,22 & 0,9465 \\
\hline 21 & Mitscherlich & $16,81^{* *}$ & 3,27 & 6,04 & 0,7311 \\
\hline CB-41-76 & Quadrático & $25,45^{* *}$ & 2,02 & 6,04 & 0,7381 \\
\hline 22 & Mitscherlich & $76,23 * *$ & $4,77^{*}$ & 3,32 & 0,9139 \\
\hline CB-53-98 & Quadrático & $114,56 * *$ & 3,04 & 3,32 & 0,9156 \\
\hline 23 & Mitscherlich & $277,19^{* *}$ & $10,32 * *$ & $2,2 I$ & 0,9622 \\
\hline $\mathrm{CB}-47-355$ & Quadrático & $416,49^{* *}$ & $6,41 * *$ & 2,21 & 0,9639 \\
\hline 24 & Mitscherlich & $39,73^{* *}$ & 3,08 & 4,85 & 0,8678 \\
\hline$C P-51-22$ & Quadrático & $60,47^{* *}$ & 1,47 & 4,85 & 0,8805 \\
\hline 25 & Mitscherlich & $214,64^{* *}$ & $13,40 * *$ & 2,13 & 0,9432 \\
\hline IAC $-48-65$ & Quadrático & $326,47^{* *}$ & $5,92 *$ & 2,13 & 0,9564 \\
\hline 26 & Mitscherlich & $100,93^{* *}$ & 1,16 & 3,31 & 0,9548 \\
\hline IAC-52-150 & Quadrático & $150,61 * *$ & 1,30 & 3,31 & 0,9499 \\
\hline 27 & Mitscherlich & $69,64^{* *}$ & 1,99 & 2,68 & 0,9289 \\
\hline NA-56-79 & Quadrático & $104,61^{* *}$ & 1,23 & 2,68 & 0,9303 \\
\hline
\end{tabular}

